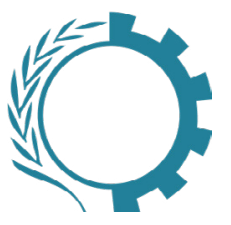

\title{
A Review on Antioxidant-rich Products as Protective and Therapeutic Agents Against Chlorpyrifos Hepatotoxicity
}

Reza Pourbabaki, Department of Occupational Health Engineering, School of Public Health, Shiraz University of Medical Sciences, Shiraz, Iran.

Sajjad Samiei, Department of Occupational Health Engineering, School of Public Health, Tehran University of Medical Sciences, Tehran, Iran.

Monireh Khadem, Department of Occupational Health Engineering, School of Public Health, Tehran University of Medical Sciences, Tehran, Iran.

Saba Kalantari, Department of Occupational Health Engineering, School of Public Health, Tehran University of Medical Sciences, Tehran, Iran.

Zahra Beigzadeh, Department of Occupational Health Engineering, School of Public Health, Tehran University of Medical Sciences, Tehran, Iran.

(1) Seyed Jamaleddin Shahtaheri, $\left({ }^{*}\right.$ Corresponding author), Department of Occupational Health Engineering, School of Public Health, Tehran University of Medical Sciences, Tehran, Iran. shahtaheri@sina.tums.ac.ir

\section{Abstract}

Background and aims: Recently, the growing concerns of the scientific community has been focused on the threat to health caused by environmental pollutants, including pesticides such as Chlorpyrifos (CPF). Therefore, finding effective ways to treat and prevent the unfavorable health effects of being exposed to such pllutants is needed. Particularly, promising for CPF, and thus arousing the greatest interest, is the possibility of using various ingredients that are present in plants, including mainly polyphenol compounds. As the liver is one of the organs that protects us against this pesticide, any disturbance in the proper functioning of this organ could lead to serious consequences for health. Thus, the aim of the present review was to discuss the possibility of using polyphenol-rich food products as a strategy for the protection against this xenobiotic hepatotoxicity and treatment of this pesticide-induced liver damage.

Methods: A comprehensive search was performed in various databases, using specific keywords such as "biologically active compounds", "antioxidants", "Chlorpyrifos/CPF", "environmental exposure", "health effects", "hepatotoxicity", "liver damage", "mechanisms of Chlorpyrifos/CPF action", "oxidative stress", "preventive strategies", "polyphenols", "protection", "risk for health", and "treatment".

Results and Conclusions: Due to the ability and the strong antioxidative potential of polyphenols, as well as their abundance in dietary products, it seems to be of high importance to consider the possibility of using polyphenols, as potential preventive and therapeutic agents against CPF hepatotoxicity, determined by its strong pro-oxidative properties. Although most of the data on the effectiveness of polyphenols, comes from animal testing, the fact that some of them are derived from experimental models reflecting human exposure to this pesticide, allows us to assume that some polyphenol food products may have promising protective effects against CPF hepatotoxicity in humans.

Conflicts of interest: None

Funding: None

\section{Keywords}

Chlorpyrifos

Liver

Hepatotoxicity

Protective effects

Oxidative stress

Antioxidants

Received: 2019/07/9

Accepted : 2020/02/3 


\section{Introduction}

Organophosphate pesticides, are the major class of agricultural chemicals, commonly used in controlling a wide range of pests in the field crops, fruits, and vegetables. Chlorpyrifos (CPF; O, O-diethyl O-3, 5, 6-trichloropyridin-2-yl phosphorothioate), is a colorless to white crystalline solid organophosphate insecticide. Due to the risks posed by chlorpyrifos to human health, the US environmental protection agency in the year 2000 placed restrictions on its domestic applications. In spite of that, chlorpyrifos is still widely used as an insecticide in agricultural practices. In fact, chlorpyrifos is currently used for the maintenance of public recreational areas. Exposure to chlorpyrifos is known to produce numerous detrimental effects, such as neurotoxicity, genotoxicity, teratogenicity, and endocrine disruption in both humans and animals. Recently, the growing attention of the scientific community has been focused on the threat to health caused by the environmental pollutants, including pesticides such as chlorpyrifos, and the need to find effective ways to treat and prevent unfavorable health effects of exposure to them. Particularly, promising for chlorpyrifos, and thus arousing the greatest interest, is the possibility of using various ingredients that are present in plants, including mainly polyphenol compounds. The available data from epidemiological studies, provide increasing evidence on the close connection between human health status and the quality of diet, as well as chemical pollution of the natural and occupational environments. These data, together with reports on the unavoidable and increasing lifetime exposure of the general population in industrialized countries to numerous substances hazardous to health, have attracted growing attention, that has now been focused not only on recognizing the main environmental risk factors for human health, but also on effective ways of preventing the unfavorable effects of exposure to them, or at least to ameliorating these effects. It is particularly important in regard to organs and systems at the highest risk of damage, such as the liver. The liver is one of the largest organs in the human body. This organ is particularly exposed to various noxious substances and poisonous products of their biotransformation, including the Free Radicals (FR) and Reactive Oxygen Species (ROS). Moreover, many xenobiotics, such as chlorpyrifos, are characterized by their ability to accumulate in the liver. Figure 1, illustrates the etiological factors of the liver injury. In mammals, a sophisticated antioxidant system has been developed to maintain the redox homeostasis in the liver (Figure 2). As the liver is one of the organs for the elimination of this pesticide from the body, and disturbances in the proper functioning of this organ has serious consequences for health, the aim of the present review was to discuss the possibility of using polyphenol-rich food products as the strategy for the protection against this xenobiotic

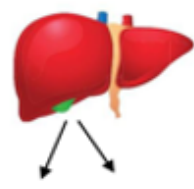

\begin{tabular}{|c|c|}
\hline Internal & External \\
\hline $\begin{array}{l}\text { medical } \\
\qquad \begin{array}{l}>\text { Reye Syndrome } \\
>\text { Inflammation } \\
>\text { Gilbert's syndrome } \\
>\text { Fatty liver disease } \\
>\text { Hemochromatosis } \\
\quad \text { A Autoimmune Hepatitis } \\
>\text { Viral infections } \\
\quad \text { Hepatitis A virus } \\
\quad \text { - Hepatitis B virus } \\
\quad \text { Hepatitis C virus }\end{array}\end{array}$ & 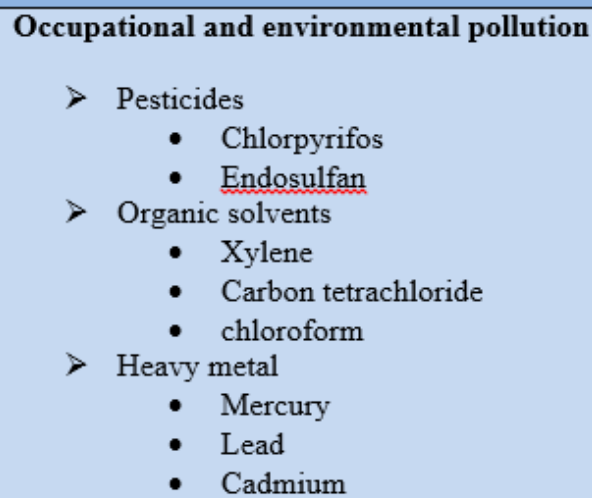 \\
\hline $\begin{array}{l}\text { Toxins } \\
\text { Mycotoxins } \\
\text { Plant toxins } \\
\text { Bacteria }\end{array}$ & $\begin{array}{l}\text { Other factors } \\
\text { Ethanol } \\
\text { Cigarette smoke } \\
\text { Amphetamine }\end{array}$ \\
\hline
\end{tabular}

Fig. 1. Etiological factors of the liver injury 


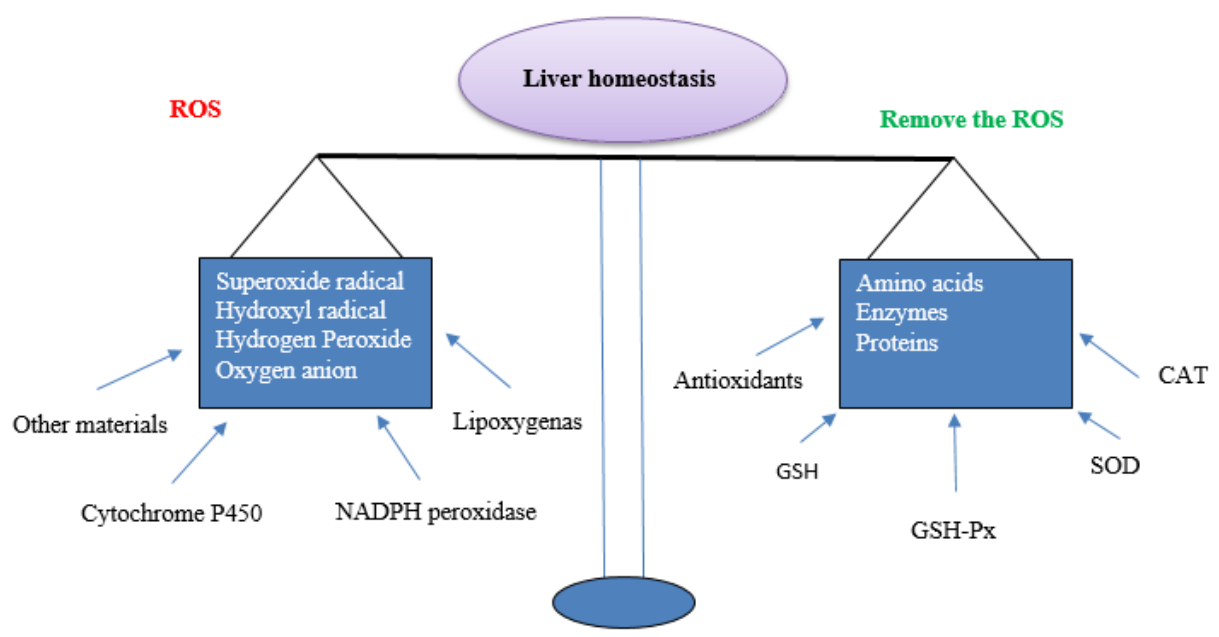

Fig. 2. Homeostasis of the liver in the body

hepatotoxicity and treatment of this pesticide-induced liver damages.

\section{Methods}

As it is necessary to obtain information about the mechanism of the liver damage caused by CPF, the pathways of this damage and useful antioxidants to reduce the effects, are presented in this article. In preparing this review, we searched for data in the bibliographic databases, such as Web of Science, ScienceDirect, Medline, Scopus, and PubMed. using keywords such as biologically active compounds, antioxidants, Chlorpyrifos/CPF, environmental exposure, health effects, hepatotoxicity, liver damage, mechanisms of Chlorpyrifos/CPF action, oxidative stress, preventive strategies, polyphenols, protection, risk for health, and treatment. Research papers published in English and Persian from 1960 to 2020 in these databases were used, and items such as editorials, letters to the editor, papers presented at conferences, and reports were excluded from the study. Keywords were selected based on the PICO principle. Then, the review process, screening and removal of duplicate and irrelevant information was performed. In addition, the resources were retrieved, and after preparing the full text of the input articles, the data was extracted.

\section{Results}

Searchers found a total of 502 studies. Based on the inclusion and exclusion criteria, a total of 158 articles were finally included in this systematic review. Studies were published from 1960 to 2020 . Most of the studies, focused on the effects of the chlorpyrifos and the damages this toxin has on the liver, kidney, testis, lungs, and brain tissue. Studies of the mechanism of chlorpyrifos toxicity on the liver tissue, as well as the study of antioxidant substances in order to protect people against liver damages caused by chlorpyrifos was investigated (Table 1). Experimental data have shown that polyphenol-rich food ingredients such as lycopene and beta-carotene, can be used for the protection against chlorpyrifos-induced toxicities. Carotenoids, are a family of fat-soluble pigments, found in many fruits and vegetables. Numerous studies have shown that carotenoids can be used to reduce oxidative stress. Lycopene, is a natural carotenoid and one of the most effective antioxidants in the carotenoids family. Cactus ingredients, green tea, and curcumin, appear to be effective in preventing and treating the liver damage. While honey, quercetin, fennel, and ziziphora oil extracts can be useful in the treatment of the damages caused by chlorpyrifos. In addition, the ingredients in curcumin and cocoa are able to improve the liver function, prior to exposure to chlorpyrifos toxin. All of the substances that were studied, as well as their effective constituents, directly protect people against liver damages, and can reduce the accumulation of this toxin in the liver.

\section{Conclusion}

Since occupational and environmental exposure to chlorpyrifos is still a serious health problem, particularly in industrialized and developing countries, it is important to have an effective strategy to be protected and treat the adverse effects of chlorpyrifos. Since the liver is a major organ in the metabolism of toxins, it is important to investigate the ways to prevent oxidative stress in this organ. It is very important to notice that polyphenols are effective in offering protection against chlorpyrifos toxicity to other organs and tissues, including the kidneys, brain, and lungs, indicating the beneficial effects of these compounds on the whole organism. Moreover, polyphenolic compounds are known to protect theliver 
Pourbabaki R, et al.

Table 1. Changes in the morphological structure of the liver caused by acute and subacute intoxication with CPF

\begin{tabular}{|c|c|c|c|c|}
\hline $\begin{array}{l}\text { Type of } \\
\text { exposure }\end{array}$ & $\begin{array}{l}\text { Dosage / duration of } \\
\text { exposure }\end{array}$ & $\begin{array}{l}\text { Exposure } \\
\text { pathway }\end{array}$ & $\begin{array}{l}\text { Animal } \\
\text { species }\end{array}$ & Morphological changes in the liver \\
\hline \multirow[t]{2}{*}{ Acute } & $\begin{array}{l}2.25,4.5 \text { and } 6.75 \\
\text { micrograms per liter / } 24 \\
\text { hours }\end{array}$ & oral & Salmon & $\begin{array}{l}\text { Hyperplasia and degeneration of liver } \\
\text { tissue }\end{array}$ \\
\hline & $\begin{array}{l}51 \text { micrograms per liter / } 24 \\
\text { hours }\end{array}$ & oral & goldfish & $\begin{array}{l}\text { Lipid peroxidation, Changes in the } \\
\text { level of activity of antioxidant enzymes }\end{array}$ \\
\hline \multirow[t]{11}{*}{ Subacute } & $\begin{array}{l}1,2,4,8 \text { and } 10 \text { Micrograms } \\
\text { per gram / } 14 \text { days }\end{array}$ & oral & Chicken & Damage and excessive size of the liver \\
\hline & $\begin{array}{l}10 \text { milligram per } \\
\text { Kilogram/ } 7 \text { days }\end{array}$ & oral & Female rats & Decreased $\mathrm{GSH}^{1}$ and $\mathrm{GST}^{2}$ activity \\
\hline & $\begin{array}{l}15 \text { and } 75 \text { Micrograms per } \\
\text { gram / } 14 \text { days }\end{array}$ & oral & Nile tilapia & $\begin{array}{l}\text { Liver damage and changes in } \\
\text { antioxidant enzyme levels, Decreased } \\
\text { GSH, Increased MDA, Damage to the } \\
\text { epithelial lining, And increase } \\
\text { interstitial space. }\end{array}$ \\
\hline & $\begin{array}{l}5 \text { milligram per } \\
\text { Kilogram / } 14 \text { days }\end{array}$ & oral & Male rats & $\begin{array}{l}\text { Increased levels of IL- } \beta 1^{3}, \mathrm{NO}^{4} \text { and } \\
\text { TNF- } \alpha^{5} \text { and inflammation of the liver }\end{array}$ \\
\hline & $\begin{array}{l}20 \text { milligram per } \\
\text { Kilogram / } 15 \text { days }\end{array}$ & oral & Male mouse & Increase $\mathrm{ALP}^{6}, \mathrm{AST}^{7}$ and $\mathrm{ALT}^{8}$ levels \\
\hline & $\begin{array}{l}r / \Upsilon \text { and } 6.2 \text { milligram per } \\
\text { Kilogram / } 28 \text { days }\end{array}$ & oral & Male rats & Changes in the liver parenchyma \\
\hline & $\begin{array}{l}1,0.1 \text { and } 0.01 \text { micrograms } \\
\text { milligram } \\
\text { per liter / } 25 \text { days }\end{array}$ & oral & Salmon & $\begin{array}{l}\text { Induction of oxidative stress and } \\
\text { changes in } \mathrm{CAT}^{9} \text { and } \mathrm{SOD}^{10} \text { enzyme } \\
\text { levels }\end{array}$ \\
\hline & $\begin{array}{l}50,100 \text { and } 200 \text { milligram } \\
\text { per } \\
\text { Kilogram / } 3 \text { days }\end{array}$ & oral & Male rats & $\begin{array}{l}\text { The decrease in Superoxide dismutase, } \\
\text { catalase and glutathione peroxidase } \\
\text { enzymes, increase in Malondialdehyde } \\
\text { levels in the liver }\end{array}$ \\
\hline & $\begin{array}{l}50 \text { and } 100 \text { milligram per } \\
\text { Kilogram / } 3 \text { days }\end{array}$ & $\begin{array}{l}\text { Intra } \\
\text { Protaneal } \\
\text { ( IP) }\end{array}$ & Male rats & Genetic toxicity and DNA damage \\
\hline & $\begin{array}{l}12 \text { and } 24 \text { micrograms per } \\
\text { liter / } 96 \text { hours }\end{array}$ & $\begin{array}{l}\text { Intramuscula } \\
\mathrm{r} \text { injection } \\
\text { (IM) }\end{array}$ & tilapia & Increase CAT and decrease SOD \\
\hline & $\begin{array}{l}3.1 \text { and } 6.2 \text { milligram per } \\
\text { Kilogram / } 28 \text { days }\end{array}$ & oral & Male rats & $\begin{array}{l}\text { Cell nucleus damage, inflammation, } \\
\text { and changes in the structure of liver } \\
\text { tissue and swelling of the cytoplasm }\end{array}$ \\
\hline
\end{tabular}

${ }^{1}$ Glutathione

${ }^{2}$ Glutathione S-transferases

${ }^{3}$ Interleukin-1 $\beta$

${ }^{4}$ Nitric Oxide

${ }^{5}$ Tumor Necrosis Factor alpha

${ }^{6}$ Alkaline phosphatase

${ }^{7}$ Aspartate transaminase

${ }^{8}$ Alanine transaminase

${ }^{9}$ Catalase

${ }^{10}$ Superoxide dismutase 
from the hepatotoxic action of other xenobiotics such as diethylnitrosamine, diazinon, and deltamethrin. It should be underlined that the beneficial properties of these compounds are widely used in the prevention and therapy of numerous diseases in humans, such as obesity, diabetes, cardiovascular diseases, and cancer. The epidemiological investigations, confirm the favorable impact of polyphenols on the liver in people with various diseases of this organ, as well as in healthy individuals. Nevertheless, further studies on this subject are needed to fully elucidate and confirm the potential prophylactic and therapeutic effects of the consumption of products rich in polyphenolic compounds, on the liver of individuals that are environmentally and occupationally exposed to chlorpyrifos. Future investigations, should focus on exploring the connection between the intake of polyphenols and these compounds, as well as the concentrations of their metabolites in the blood and urine, and the chlorpyrifos concentration in these biological fluids in relation to the liver status, evaluated based on the measurement of biomarkers of this organ damages. Experimental studies show, that the polyphenol-rich dietary products, may be effective factors in the protection against chlorpyrifos-induced liver injuries. Due to the lack of specific therapy for chlorpyrifos poisonings, polyphenolic compounds are promising agents that may be used not only in the prevention but also in the treatment of this pesticide intoxication in humans. Thus, it is necessary to evaluate the relation between the liver status and consumption of polyphenols by humans and to find the most effective and safe method to counteract the negative effects of being exposed to this toxic. Although, further studies need to be done on this subject, based on the results of the experimental investigations, including our own experimental findings, which were discussed in the present review, it can be concluded that, the use of polyphenol-rich products may be a good strategy for prevention and treatment of liver damages in humans exposed to chlorpyrifos.

\section{Conflict of interest}

The authors declare that there are no conflicts of interest regarding the publication of this manuscript.

\section{How to cite this article:}

Reza Pourbabaki, Sajjad Samiei, Monireh Khadem, Saba Kalantari, Zahra Beigzadeh, Seyed Jamaleddin Shahtaheri. A Review on Antioxidant-rich Products as Protective and Therapeutic Agents Against Chlorpyrifos Hepatotoxicity. Iran Occupational Health. 2021 (01 May);18:9.

*This work is published under CC BY-NC- 4.0 licence 

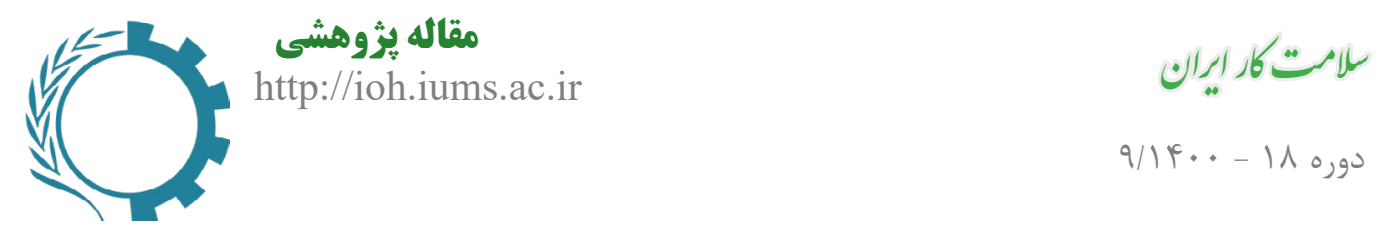

$q / 14 \ldots-110,0$

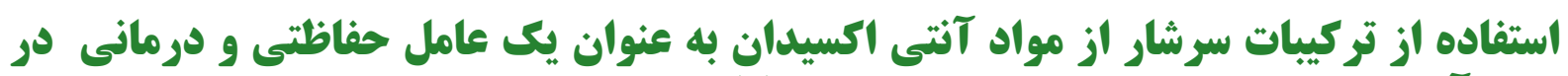

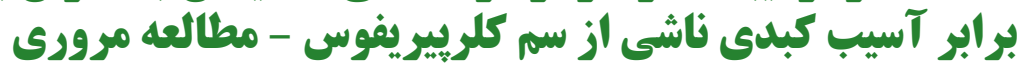

رضا يوربابكى: دانشجوى دكترى تخصصى، كروه مهندسى بهداشت حرفه ای، دانشكده بهداشت، دانشكاه علوم يزشكى شئى شيراز، شيراز، ايران.

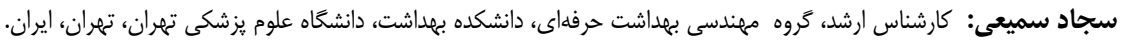

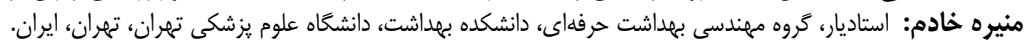

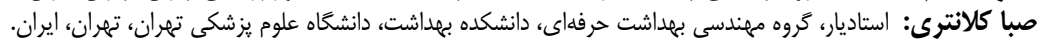

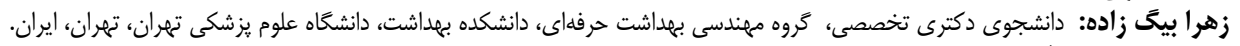

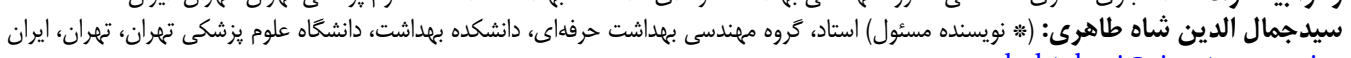
shahtaheri@sina.tums.ac.ir.



شيوه استناد به اين مقاله:

Reza Pourbabaki, Sajjad Samiei, Monireh Khadem, Saba Kalantari, Zahra Beigzadeh, Seyed Jamaleddin Shahtaheri. A Review on Antioxidant-rich Products as Protective and Therapeutic Agents Against Chlorpyrifos Hepatotoxicity. Iran Occupational Health. 2021 (01 May);18:9. 
<smiles>CCOP(=S)(OCC)Oc1nc(Cl)c(Cl)cc1Cl</smiles>

شكل ا. ساختار كلرييريفوس

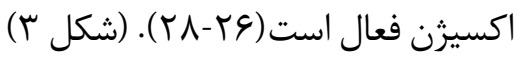

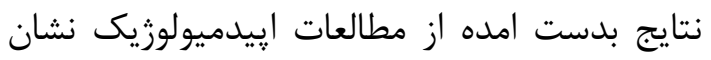

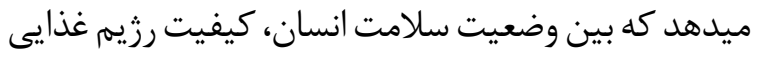

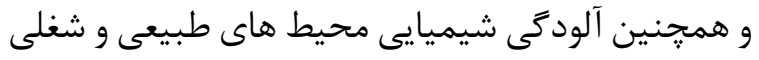

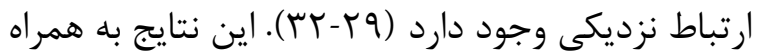

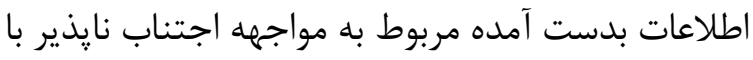

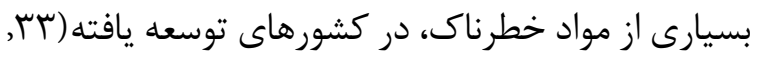
دF (MF

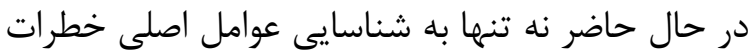

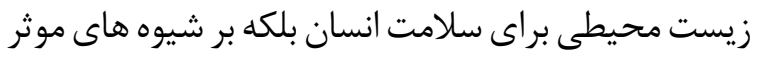

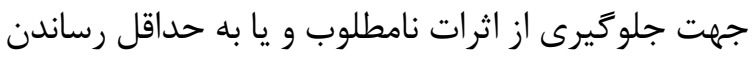

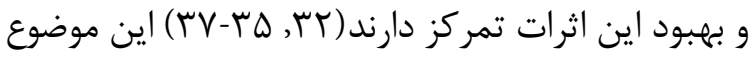

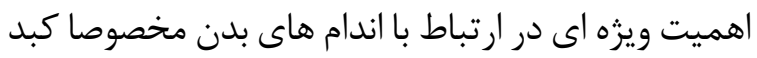

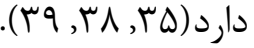

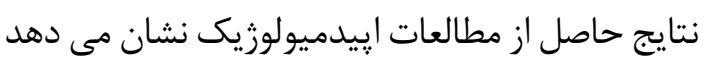

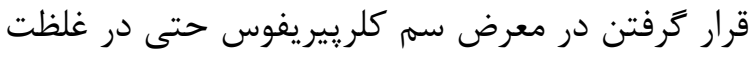

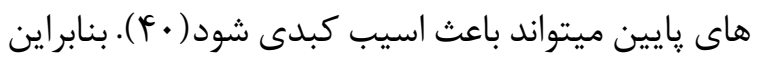

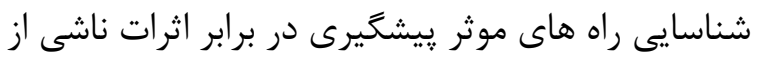

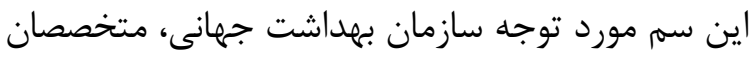

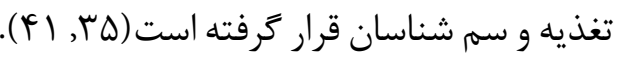

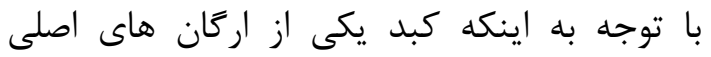

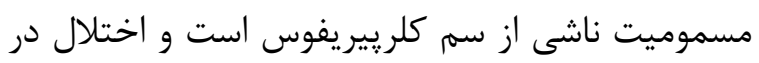

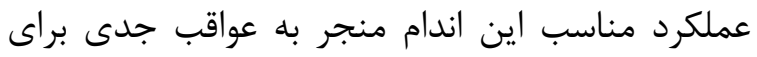

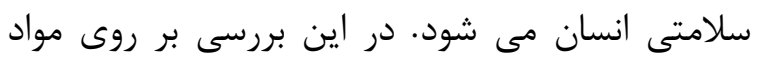

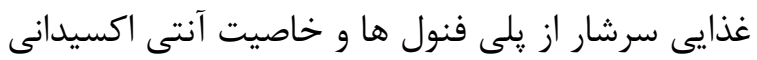

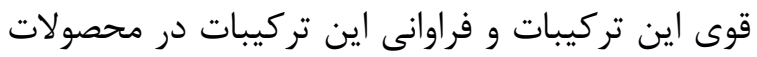

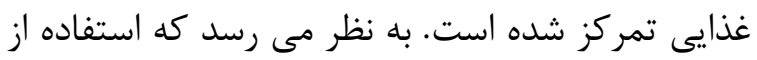

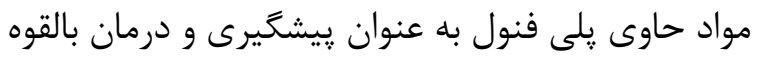

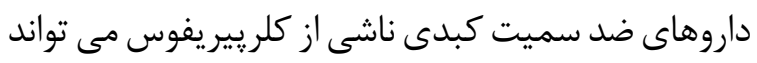

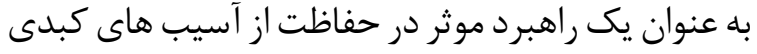
ناشى از سم كلرييريفوس مورد استفاده قرار گيرد.

روش بررسى همانكَونه كه كسب اطلاعات در خصوص مكانيسم

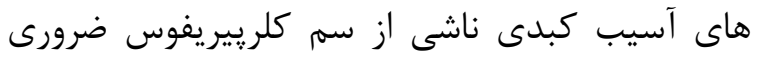

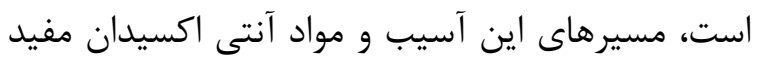

مقدمه

آفت كش هاى ار كانوفسفره اصلى ترين دسته از سموم

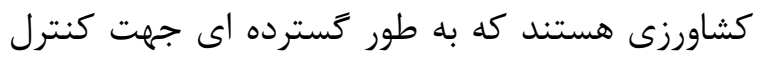

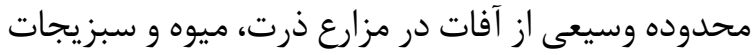

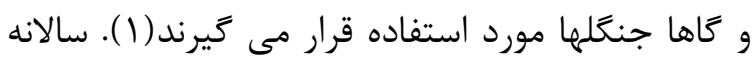

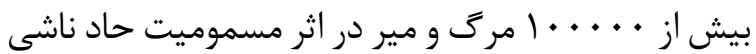

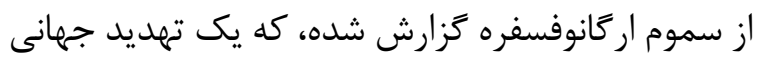

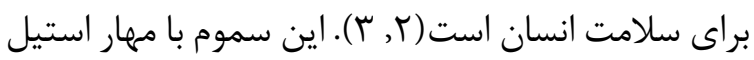

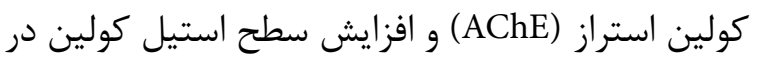

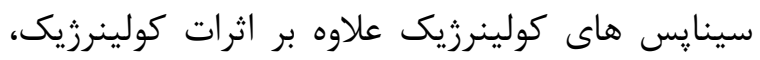

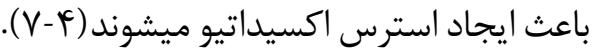

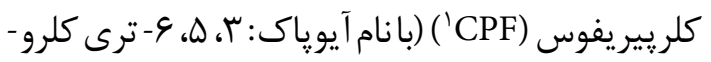

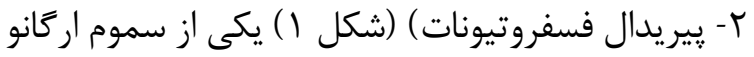

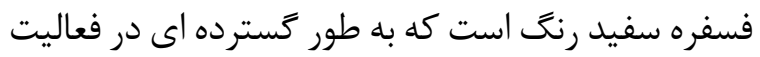

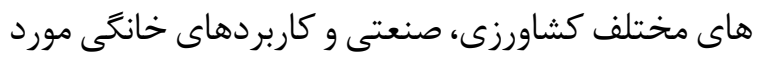

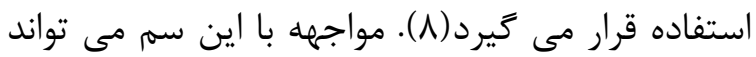

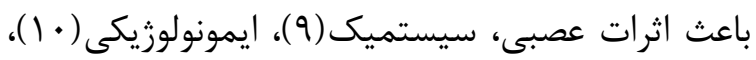

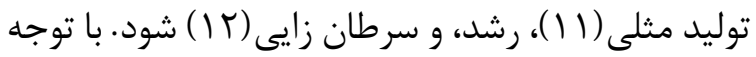

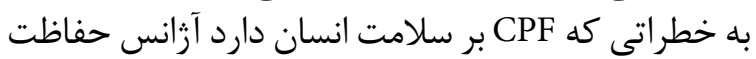
از محيط زيست آمريكا (EPA

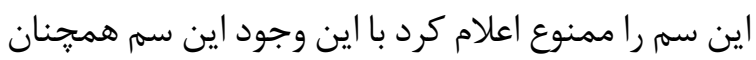

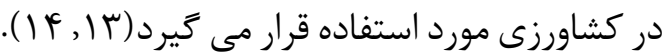

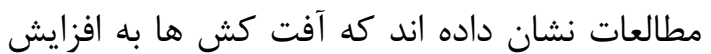

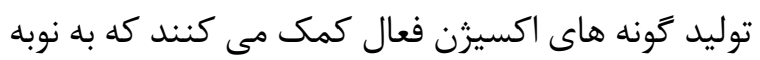

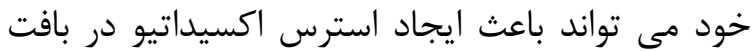

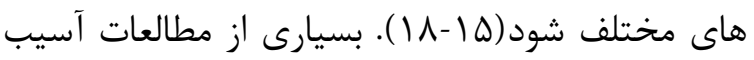

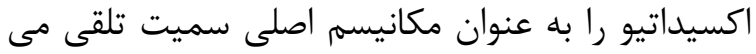

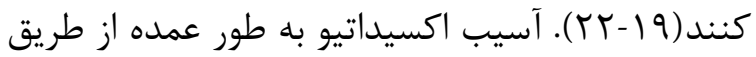

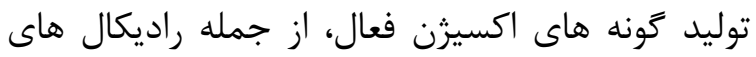

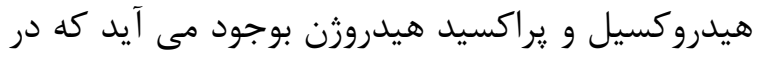

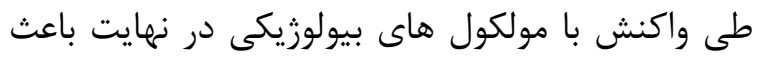

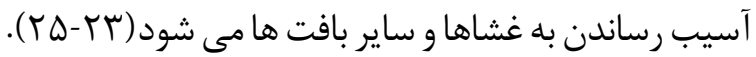

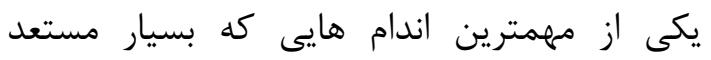

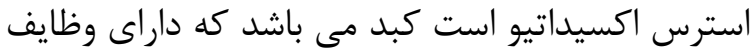

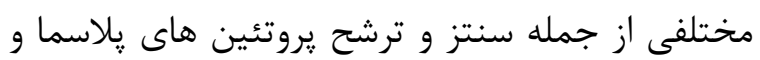

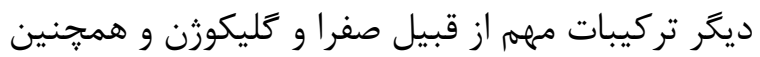

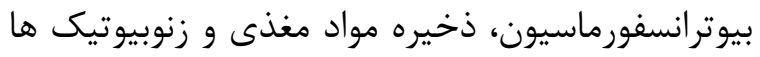

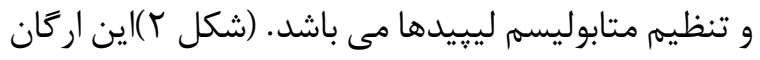

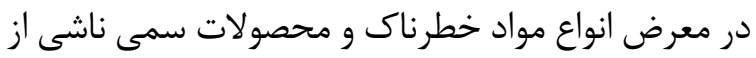

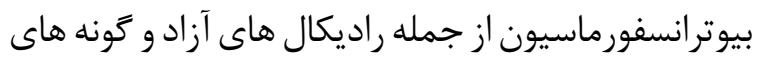

1 Chlorpyrifos

2 Environmental Protection Agency

Iran Occupational Health. 2021 (01 May);18: 9. 

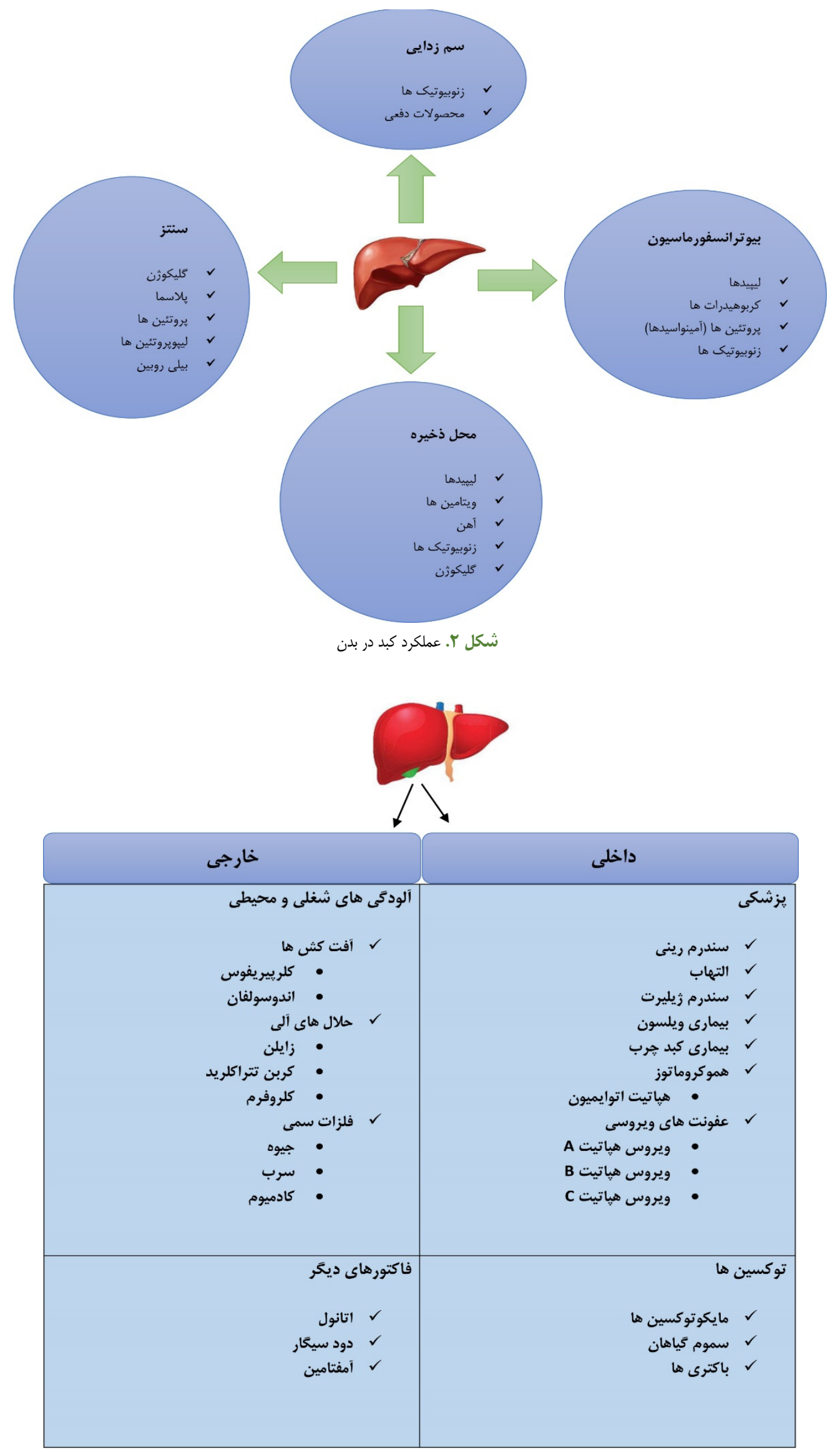

شكل "ا. فاكتورهاى موثر در ايجاد آسيب كبدى 


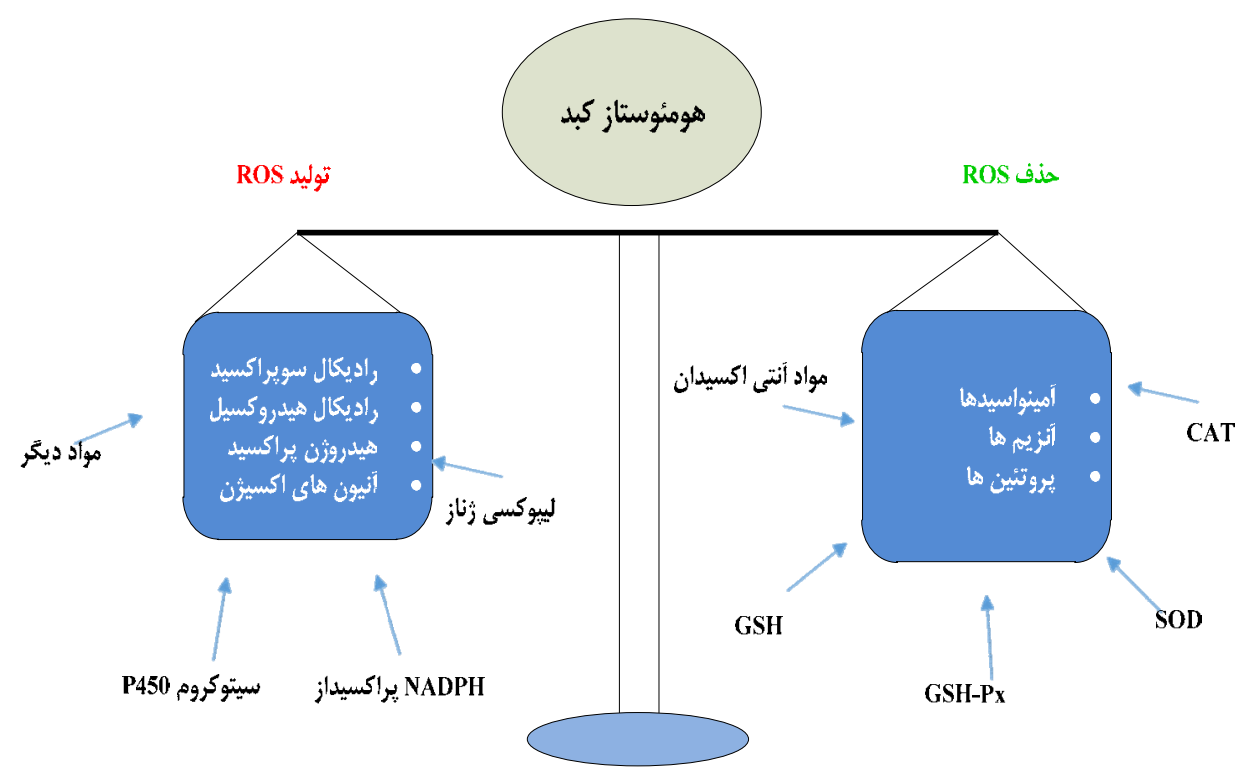

شكل ع. هومئوستاز كبد در بدن

صورت گرفته بود. در مطالعات ورودى به مكانيسم سميت

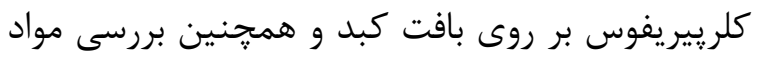

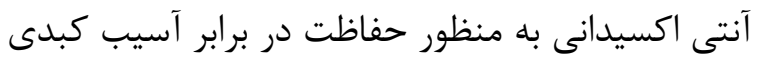
ناشى از كلرييريفوس يرداخته شده است إنـ

1 - كبد يكى /ز /ندام هاى اصلى به منظور تجمع و سميت

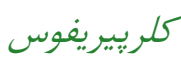
كبد نقش بسيار مهمى در متابوليسم روزانه فرد و

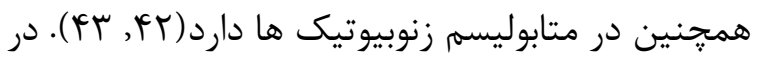

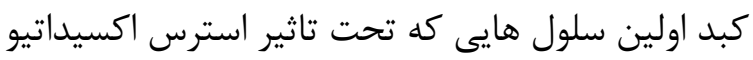

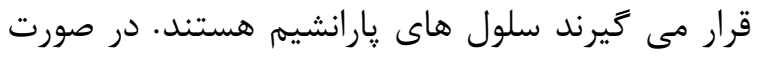

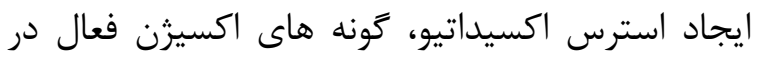

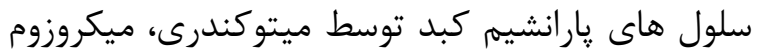

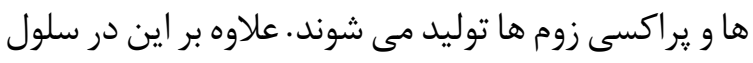

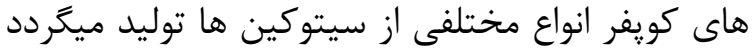

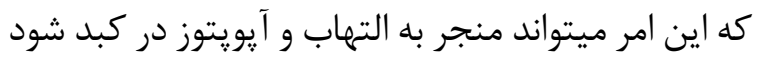

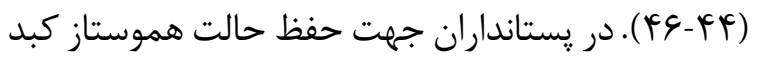

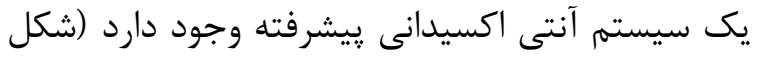

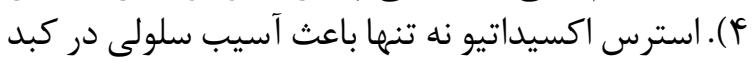

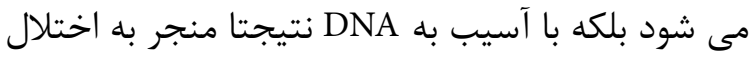

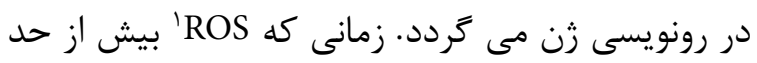

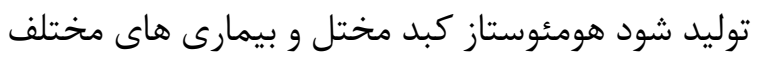

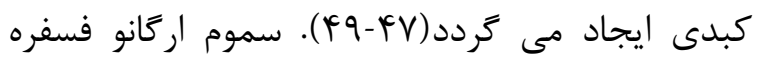

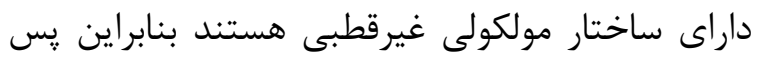
از جذب به سرعت مى توانند در كبد، غده هاى هولى بزاقى

1 Reactive Oxygen Species
جهت كاهش اثرات كبدى، در مقاله حاضر ارائه شده

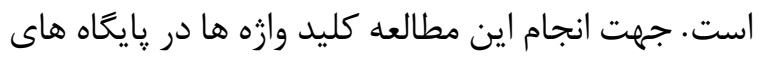
داده اي مانند Web of Science، Medline ، PubMed و Scopus، ScienceDirect كرفتند. كلمات كليدى مورد بررسى شامل آنتى اكسيدان،

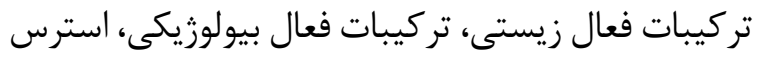

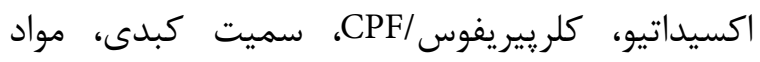

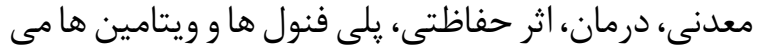

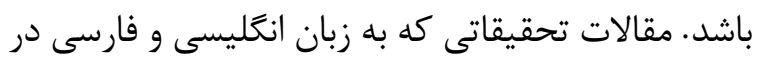

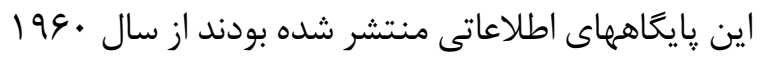

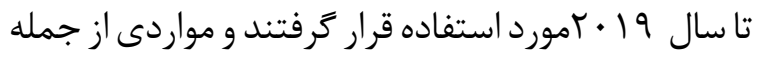

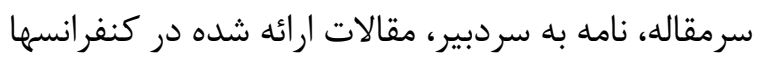

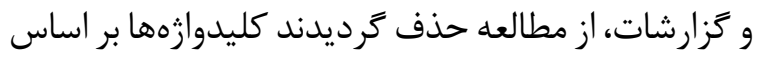

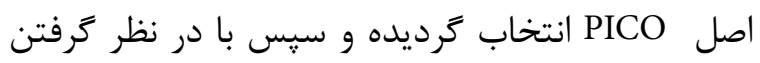
معيارهاى ورود و خروج، فرايند مرور، غربال گرىى و حذف إنف

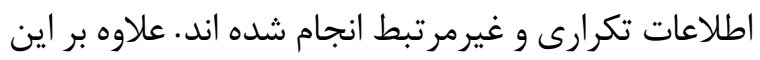

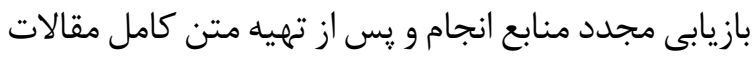
ورودى ، استخراج داده ها انجام ترديد.

يافته ها

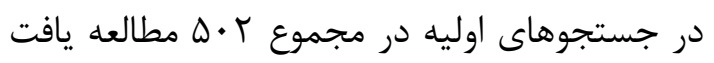

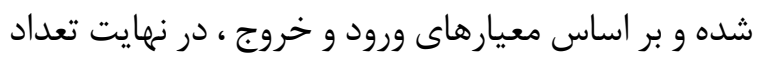

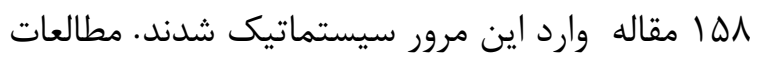

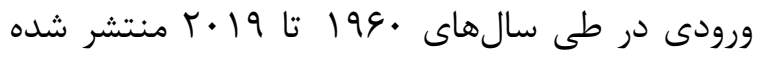

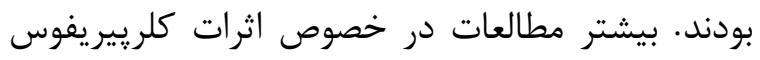
و آسيب اين سم به بافت كبد، كليه، بيضه، ريه ائرات وكرير مغز 
به خودى خود مهار كننده قوى آنزيم AchE نمى باشد ولى تبديل شدن آن به فرم اكسون (CPF-oxon) (شكل آنس

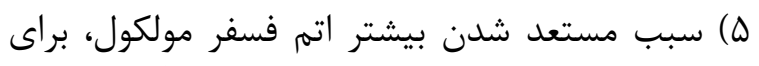

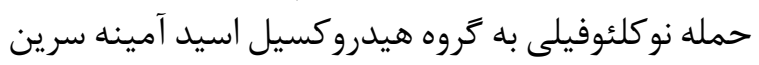

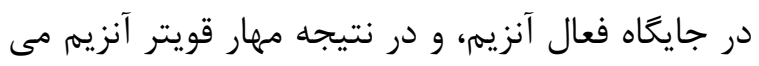

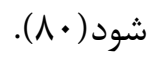

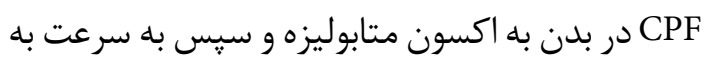

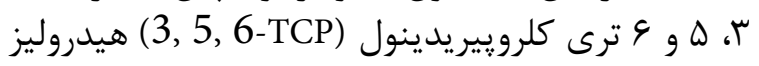

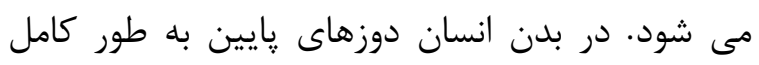

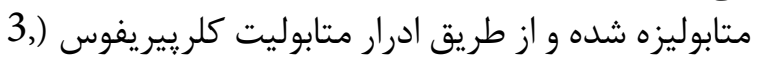

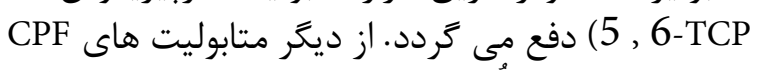

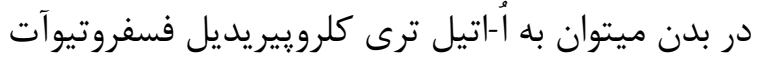
اشاره (O-ethyl trichloropyridyl phosphorothioate) كرد كه از طريق كنزو گاسيون كلوتاتيون از بدن دفع مى

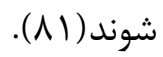
كلرييريفوس در بدن توسط سيستم سيتوكروم P450)

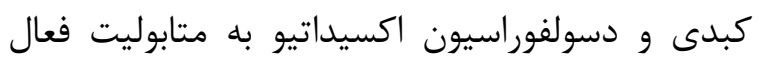

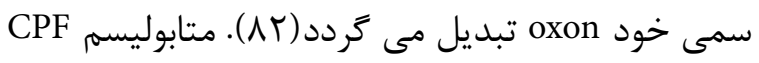

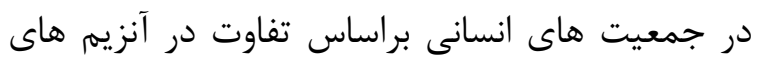

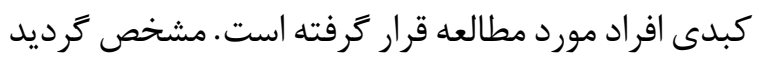

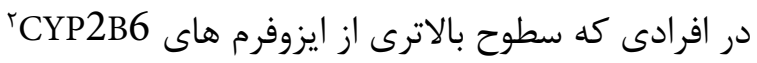

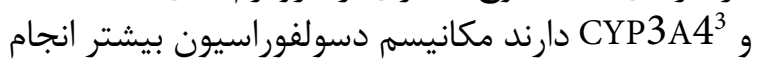

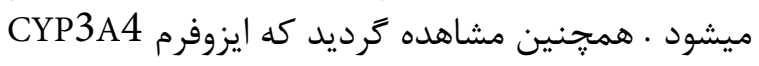

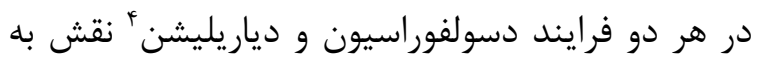

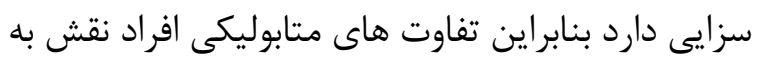

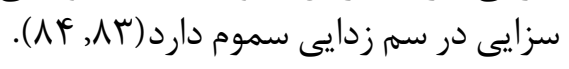

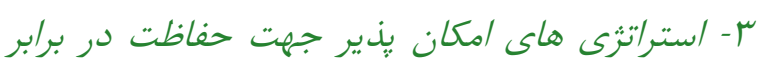

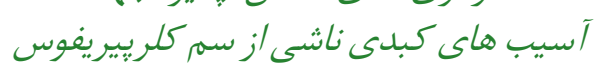

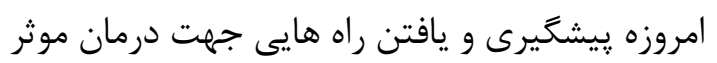

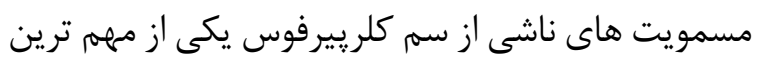

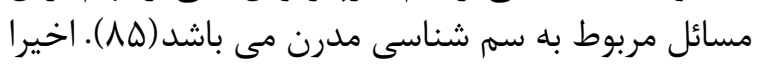

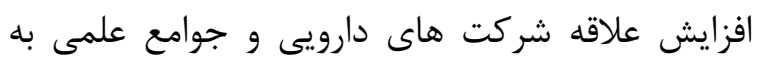

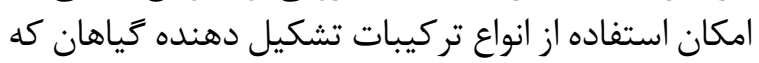

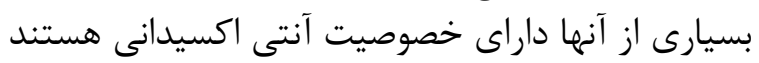

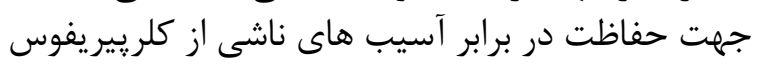

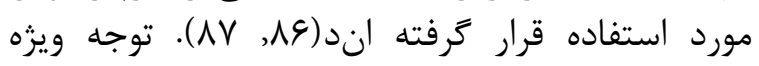

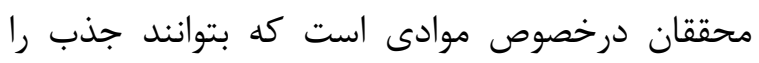

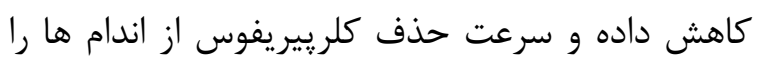

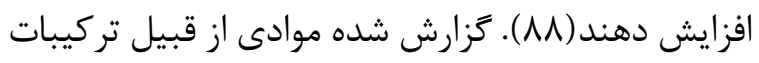

2 Cytochrome P450 2B6

3 Cytochrome P450 3A4

4 dearylation

Iran Occupational Health. 2021 (01 May);18: 9

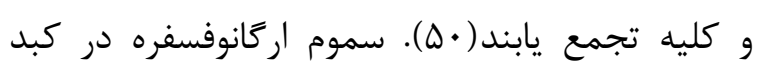

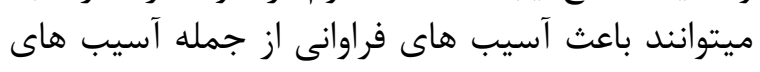

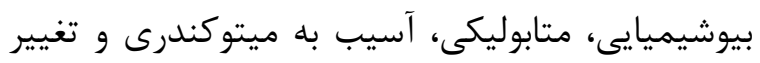

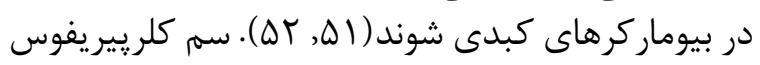

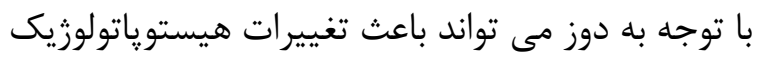

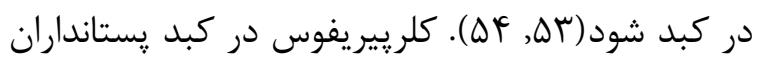
توسط سيستم سيتوكروم

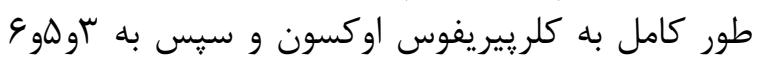

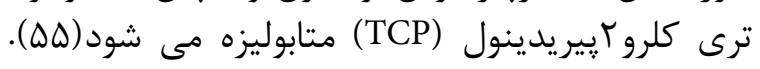

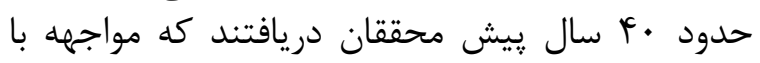

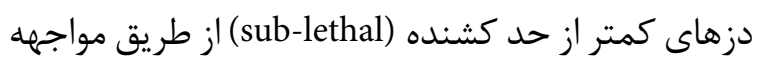

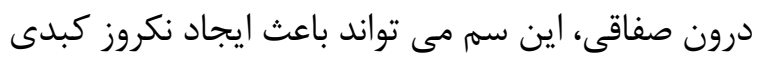

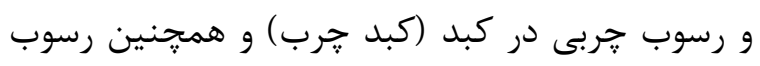

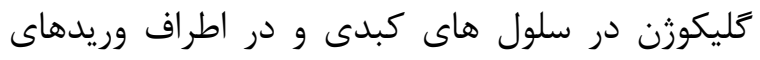

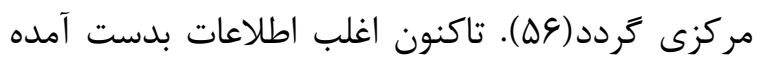

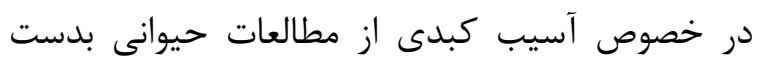

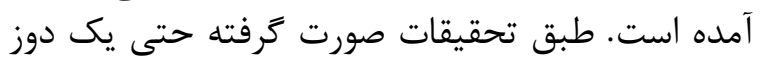

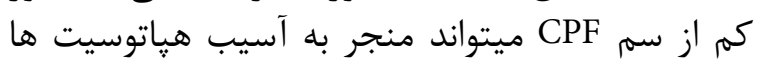

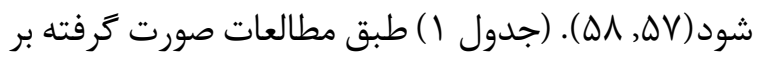

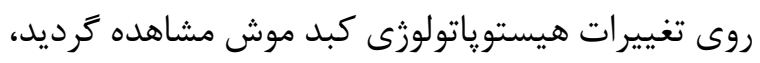

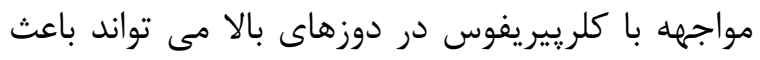

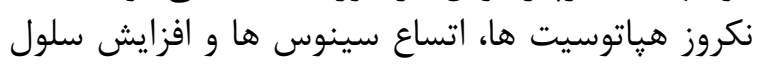

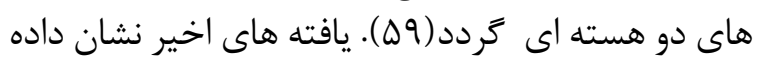

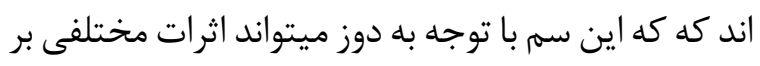

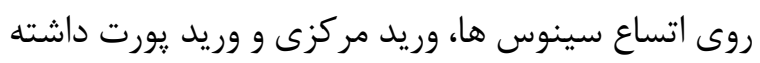
باشد •(9).

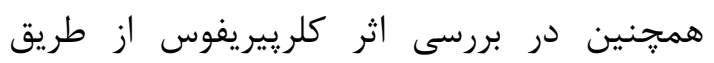

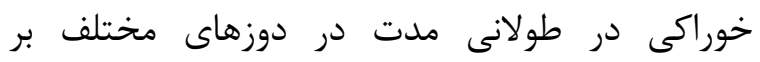

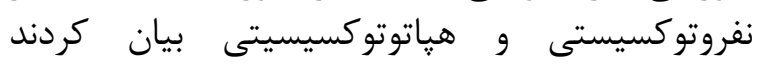

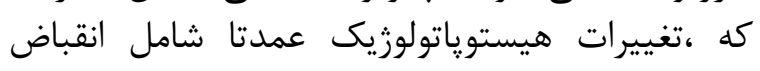

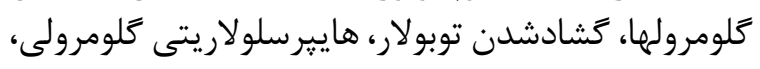

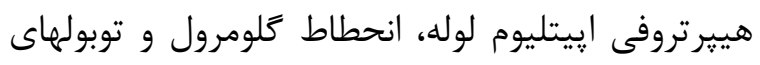

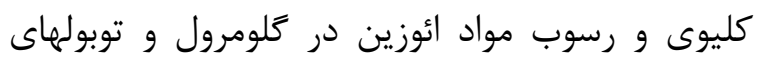

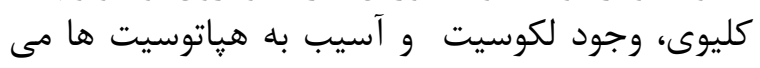

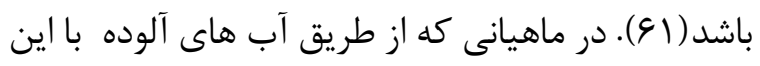

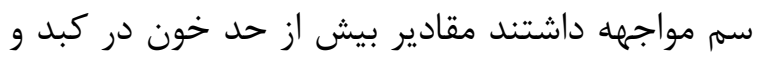

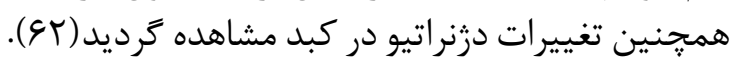

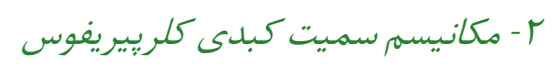

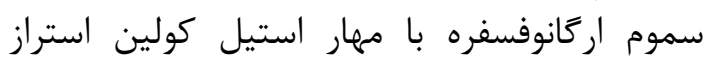

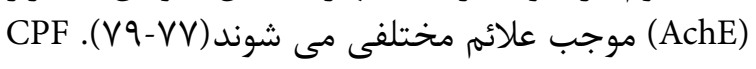


استفاده از تركيبات سرشار از مواد آنتى اكسيدان ....

جدول (. تغييرات در ساختار مورفولوزيكى كبد به دليل سميت ناشى از كلرييريفوس

\begin{tabular}{|c|c|c|c|c|c|}
\hline 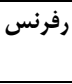 & تغييرات مورفولوزيكى در كبد & تحونه حيوانى & راه مواجهه & 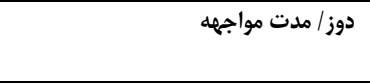 & 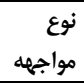 \\
\hline$(q 4)$ & هاييريلازى و درثنراسيون بافت كبد & ماهى قزل آلا & 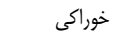 & 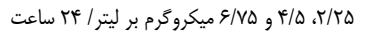 & \\
\hline$(\$ \%)$ & اكسيدان يراكسيداسيون يييدى، تغيير در سطح فعاليت آنزيم هاى آنتى & ماهى قرمز & 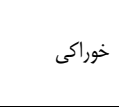 & 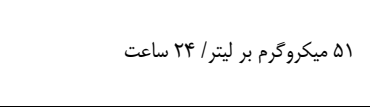 & حاد \\
\hline$(\kappa \cdot)$ & 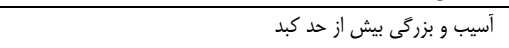 & جوجه & خوراكى & 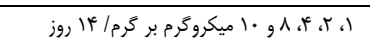 & \\
\hline$\left.(\$)^{*}\right)$ & 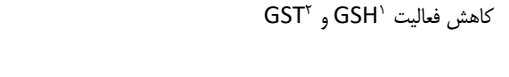 & ماده محرايى & 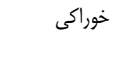 & 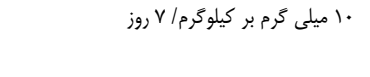 & \\
\hline$(\& \Delta)$ & 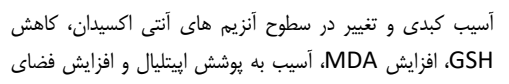 & نماهى تيلاييلاى & 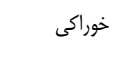 & 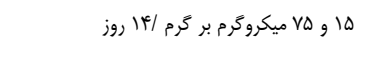 & \\
\hline$(99)$ & 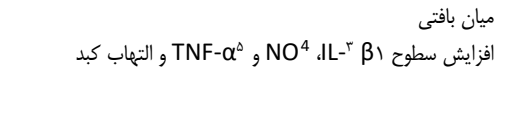 & ن موش صحرايى & 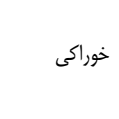 & هميلى كرم بر كيلوكرم/ أ| روز & \\
\hline$(\$ \vee)$ & 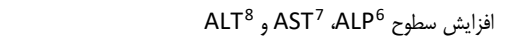 & 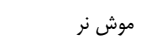 & 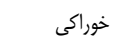 & 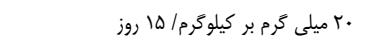 & \\
\hline$(\varsigma \cdot)$ & ت تغيير در يارارانشيم كبد & 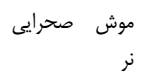 & 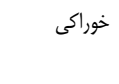 & 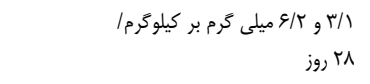 & تحت \\
\hline$(\$ \wedge)$ & القا استر اكسيداتيو و تغيير در سطوح آنزيمهاى CAT و & ماهى قزل آلا & 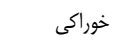 & 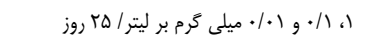 & حاد \\
\hline$(\$ 9)$ & 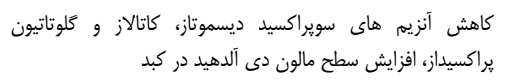 & 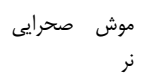 & تززيق صفاقى & 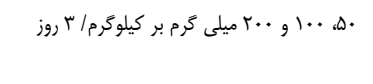 & \\
\hline$(v \cdot)$ & سميت زنتيكى و آسيب به DNA & 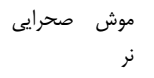 & ت ت عضلانى & له و .. اميلى كرم بر كيلوكرم/ بروز & \\
\hline (v) & افزاي CAT و كاهش SOD & ماهى تيلاييا & 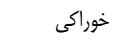 & كا و و ميكروكرم بر ليتر / وq ساعت & \\
\hline (६.) & سيتويبل به هسته سلول ها، التهاب و تغيير در ساختار بافت كبد و تورم & 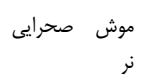 & 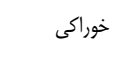 & $j g, r \Lambda / m g / k g \& / r, r / l$ & \\
\hline$(V T)$ & 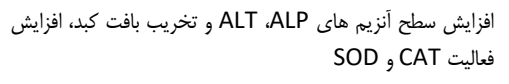 & ماهى كيور & 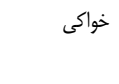 & |ه|ه/ ميلى كرم در ليتر/ / روز & \\
\hline$(\Delta 9)$ & نهاى دوقلو تخريب هياتوسيت ها، انقباض سينوس ها، افزايش سلول & ن موش صحرايى & 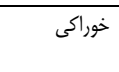 & $\begin{array}{r}\mathrm{mg} / \mathrm{kg} \mid \mathrm{r} / \mathrm{\Delta} \\
\text { A هis }\end{array}$ & \\
\hline$(\Delta F)$ & آمينيير درا آنزفيم هاز سرمى كبد (آلانين آمينو ترانسفراز و آسيارتات & 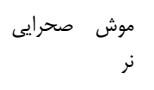 & 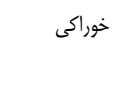 & ه/ آميلى كرم بر كيلوكرم/ مهفته & \\
\hline$\left(V^{r}\right)$ & MDA تغيير در فعاليت آنزيم هاى آنتى اكسيدان، افزايش سطح & معمولى كيور & 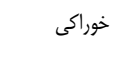 & 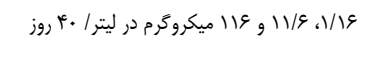 & 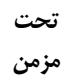 \\
\hline$\left(v^{c}\right)$ & تغيير در سيستم كبدى-صفراوى & منرش صحرايى & 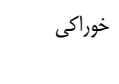 & 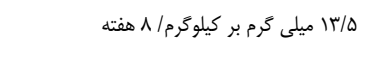 & \\
\hline$(v \Delta)$ & ها و ونكروز آنها & 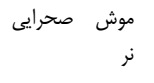 & 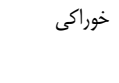 & هو •ا ميلى كَرم بر كيلوكرم/1 هفته & \\
\hline (va) & 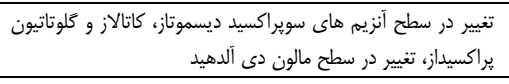 & ن موش صحرايى & خوراكى & ها ميلى كرم بر كيلوكرم/ اله هفته & 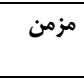 \\
\hline
\end{tabular}

${ }^{1}$ Glutathione

${ }^{2}$ Glutathione S-transferases

${ }^{3}$ Interleukin-1 $\beta$

${ }^{4}$ Nitric Oxide

${ }^{5}$ Tumor Necrosis Factor alpha

${ }^{6}$ Alkaline phosphatase

${ }^{7}$ Aspartate transaminase

${ }^{8}$ Alanine transaminase

${ }^{9}$ Catalase

${ }^{10}$ Superoxide dismutase 


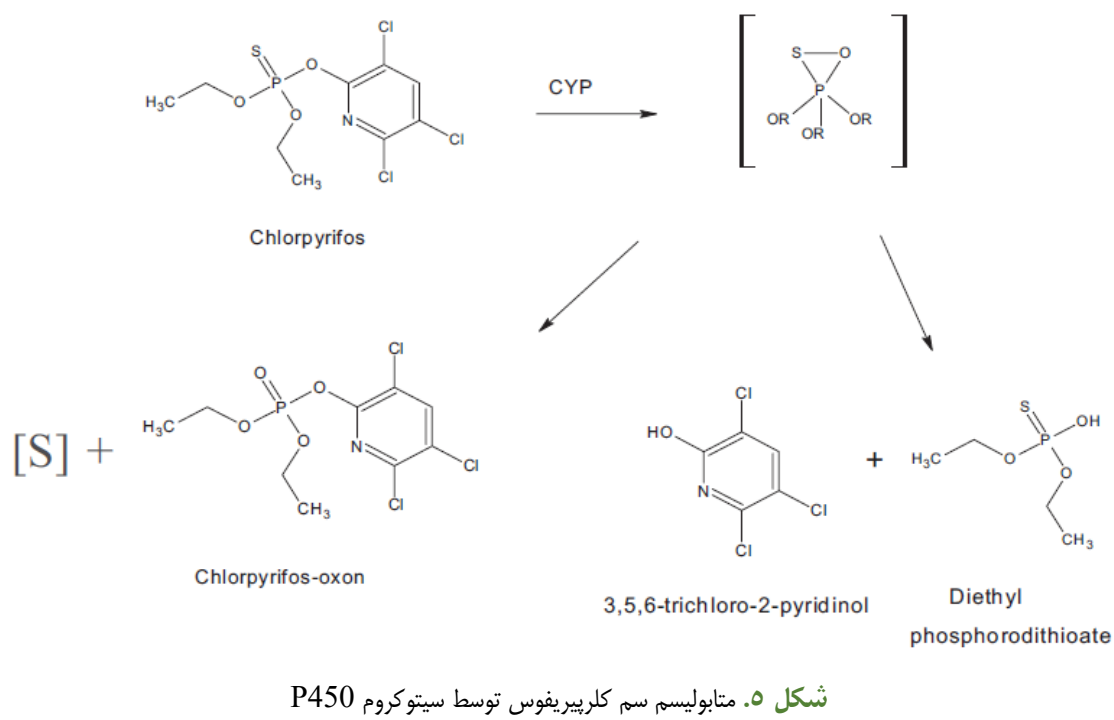

سبزيجات، روغن زيتون، جاى، قهوه، دار جين وجود دارند.

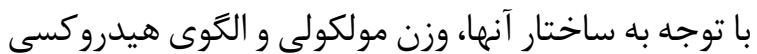

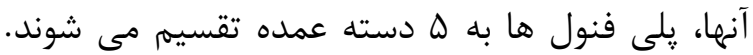

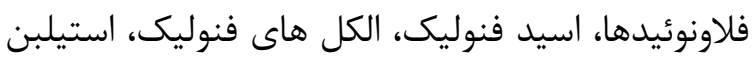

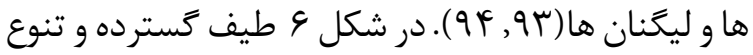

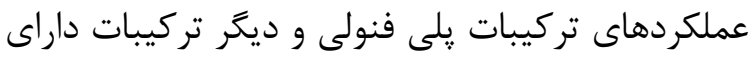

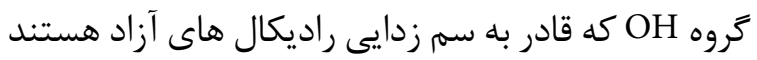

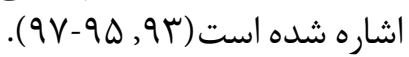

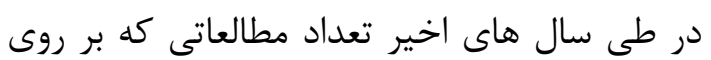

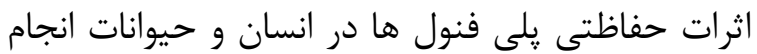

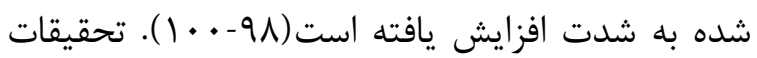
متعددى نشان مى دهند كه تركيبات يلى يلى فنول ممكن

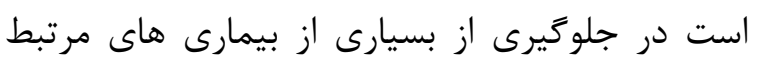

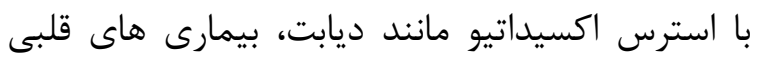

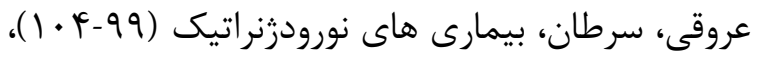

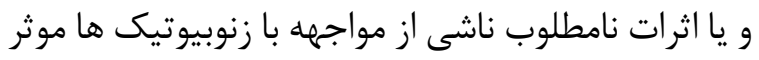

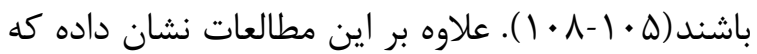

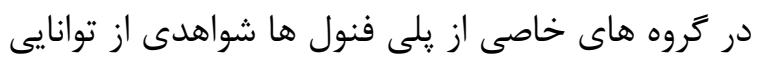

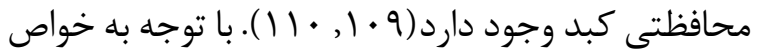

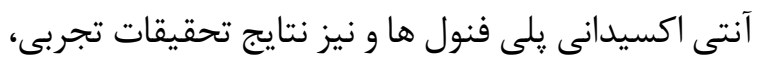

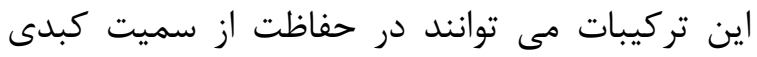

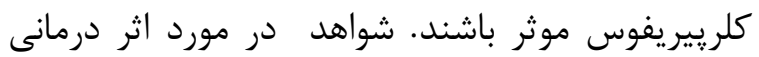

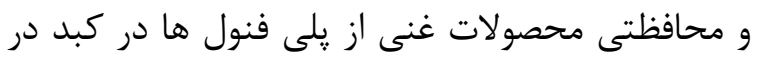

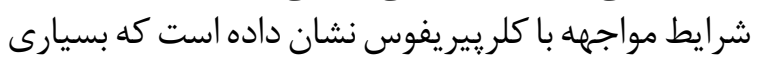

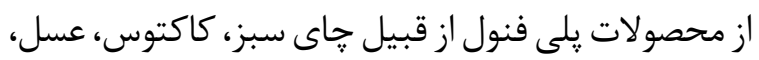

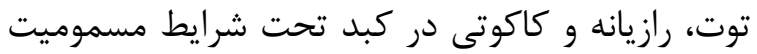

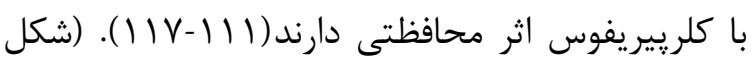

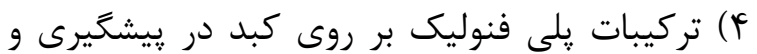

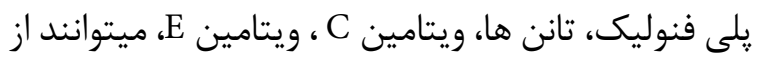

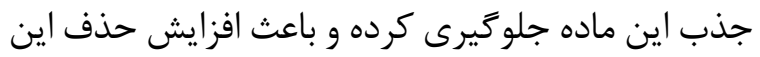

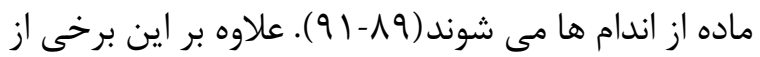

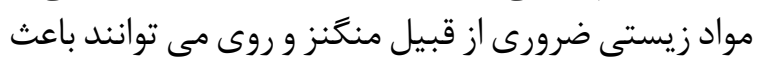

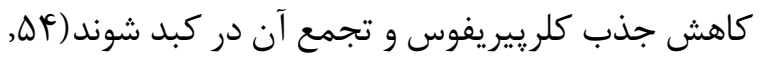

. (97, 94

با اشاره بر اين واقعيت كه مكانيسهم اصلى سميت

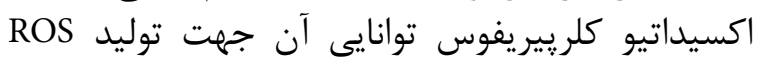

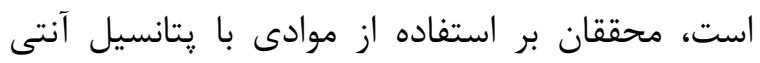

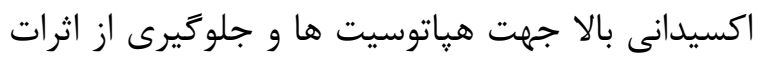

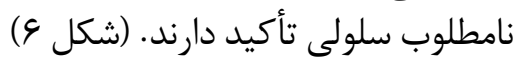

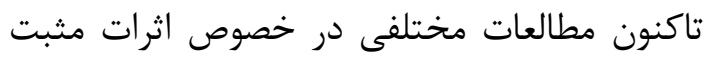

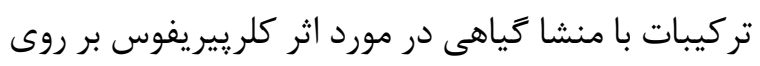

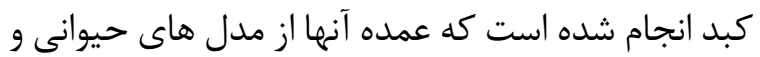

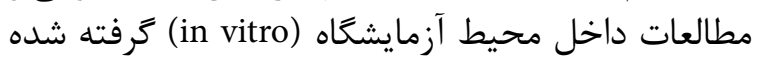

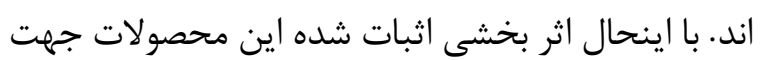

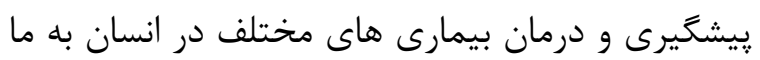

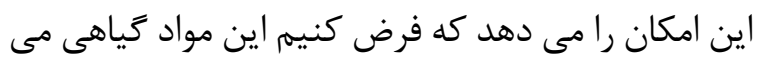

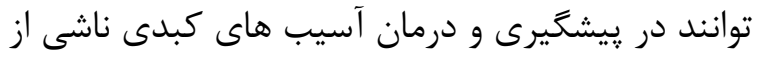

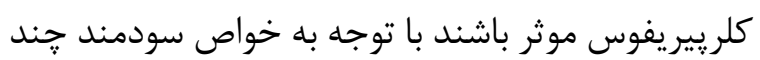

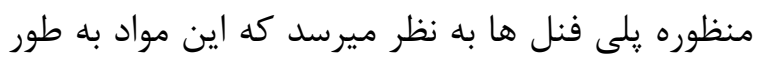

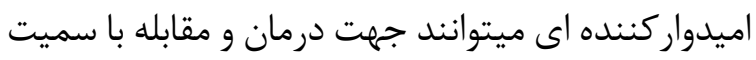
كبدى ناشى از كلرييريفوس مورد استفاده قراند

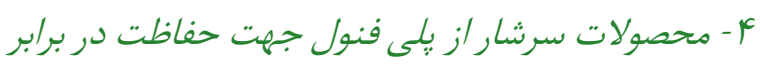

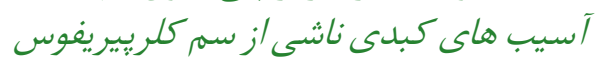

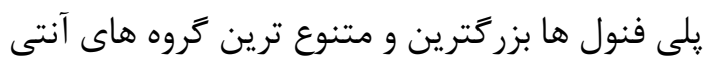

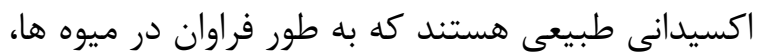




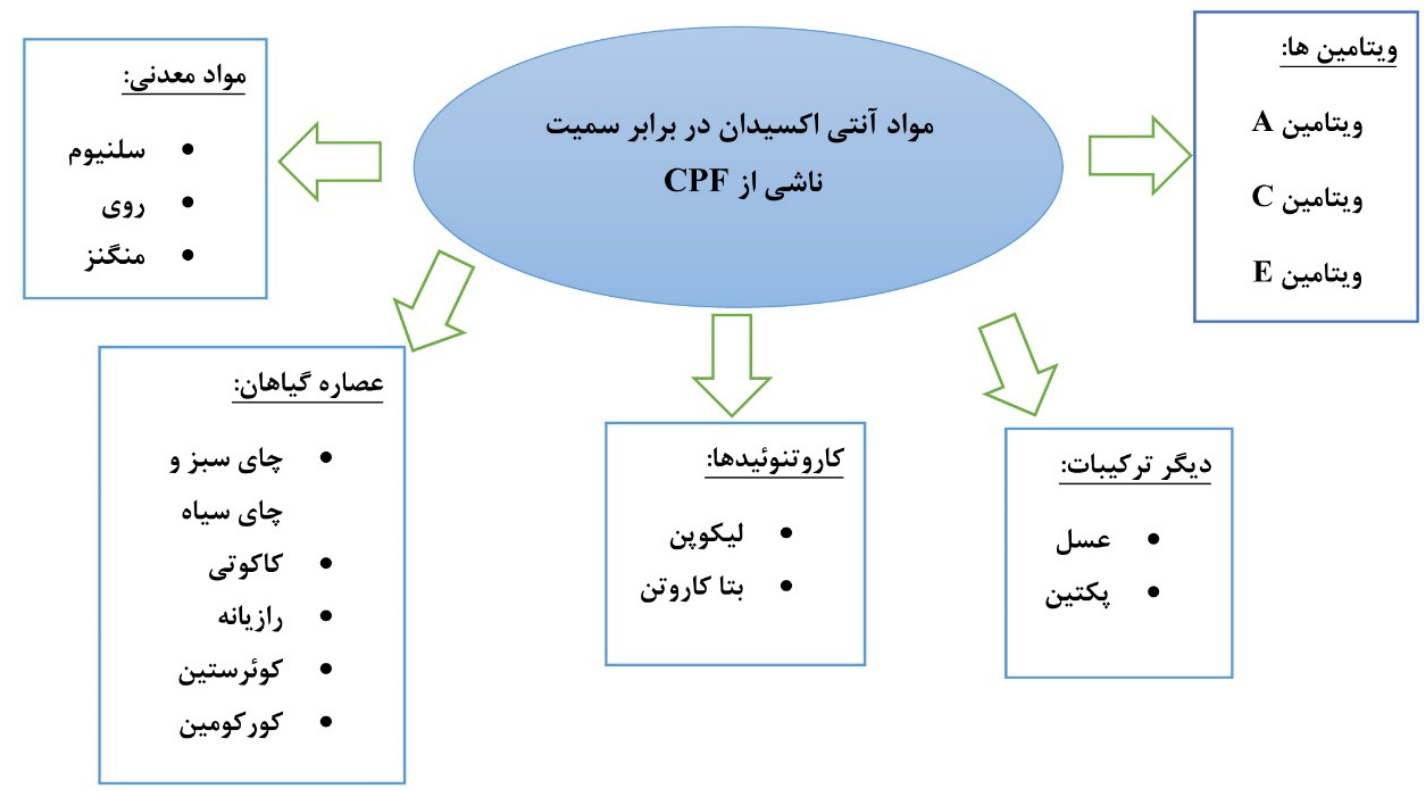

شكل 7. مواد موثر جهت حفاظت كبد در برابر كلريبريفوس

جاى سبز ميتواند در ريشخَيرى از بيمارى هاى مرتبط با

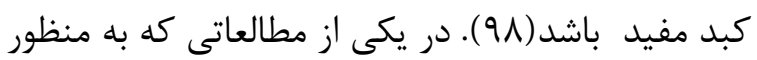

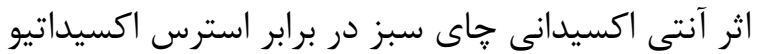

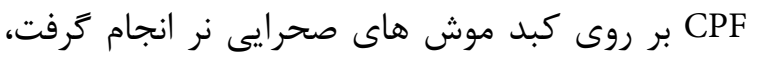

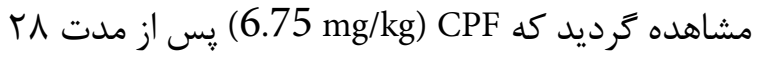

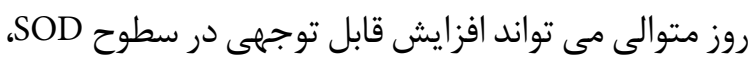

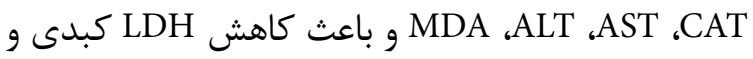
CPF شود. همجنين در موشهاى مواجها يافته

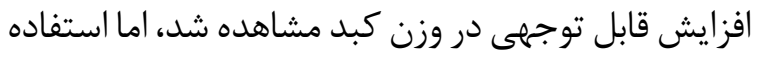

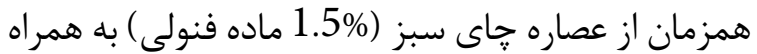

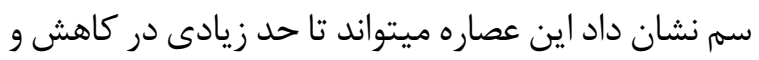

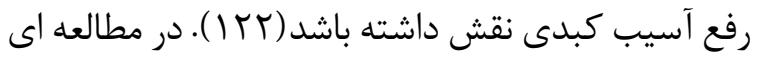

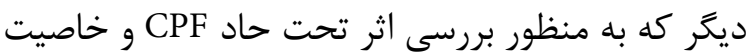

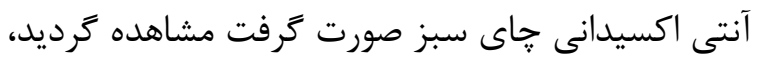

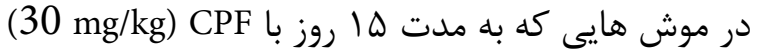

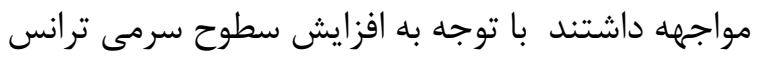

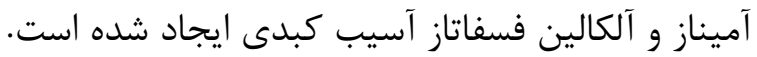

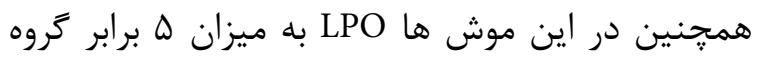

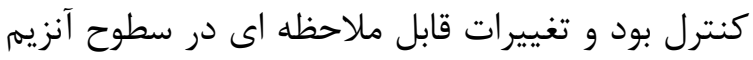

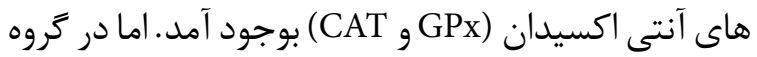

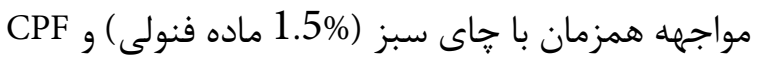

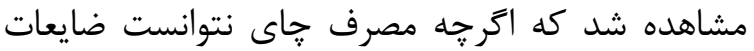

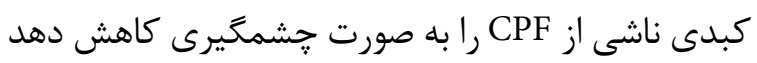

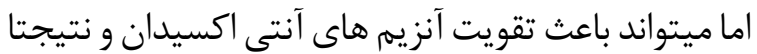
كاهش آسيب اكسيداتيو شود( (1) (1).
درمان بيمارى هاى مختلف كبد داراى اثرات مطلوبى ميى إنى

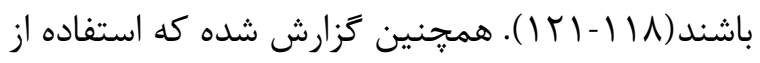

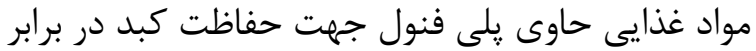

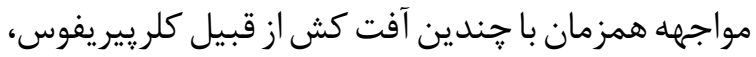

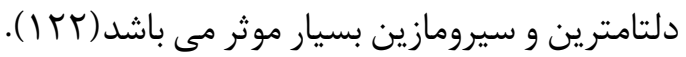

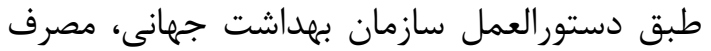

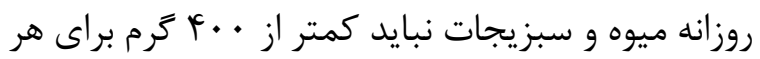

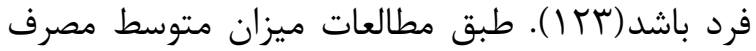

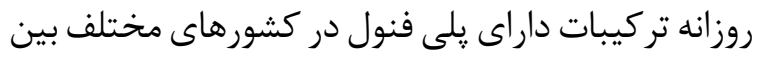

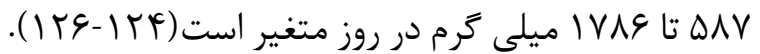

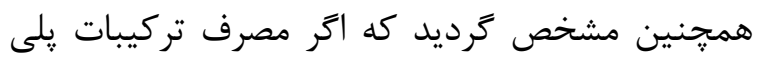

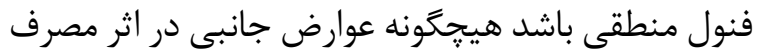

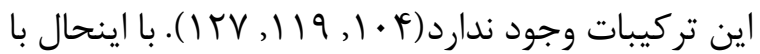

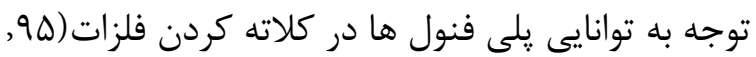

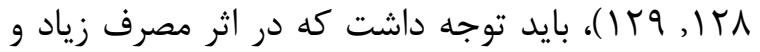

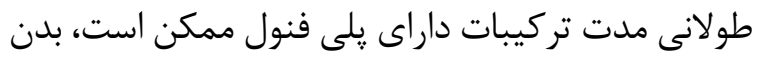

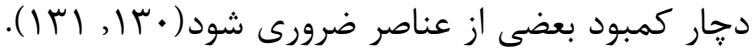

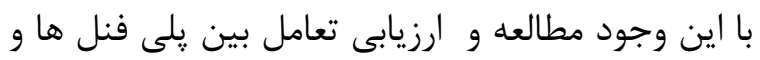
عناصر ضرورى بدن مورد نياز مى باشد.

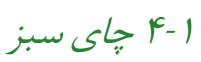
بسيارى از مطالعات نشان داده اند كه عصاره جاى سبز و

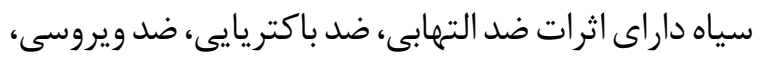

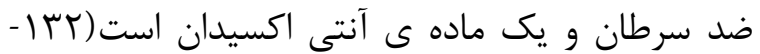

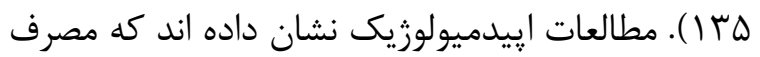


و اختلالات دستخاه گوارش توصيه ميشود(FF) (IF). در

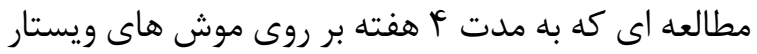

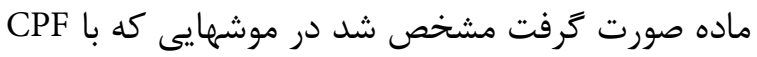

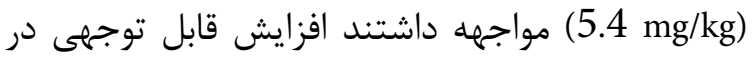

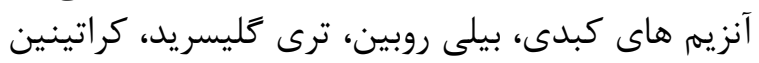

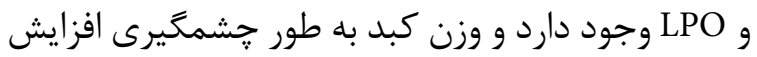

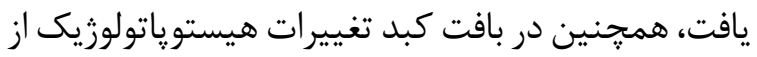

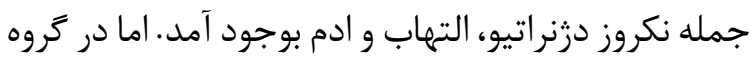

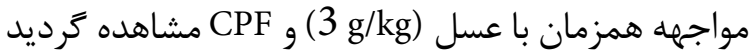

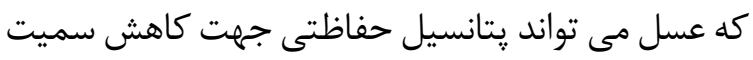
كبدى ناشى از CPF داشته باشد (1 أ (1).

\section{(Quercetin) كوئرستين}

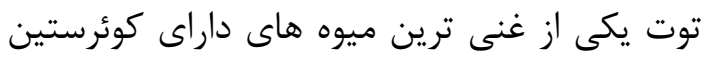

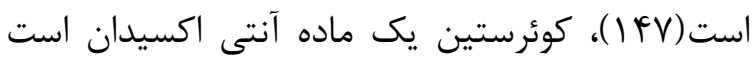

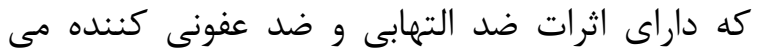

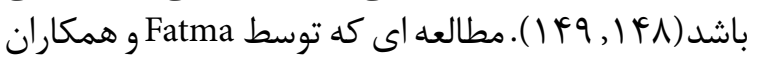

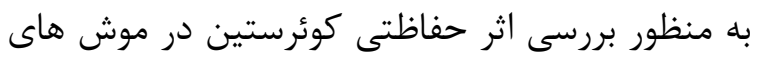

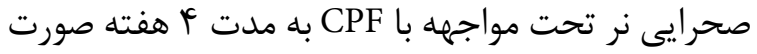

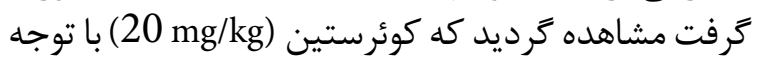

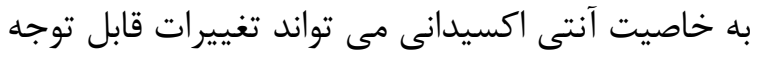

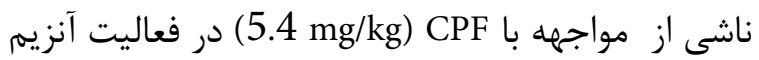

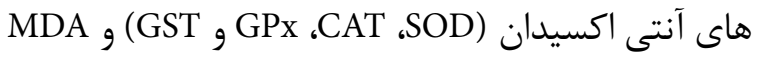

را به حالت نرمال نزديك كند (هان (1)، (CAT).

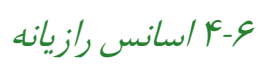

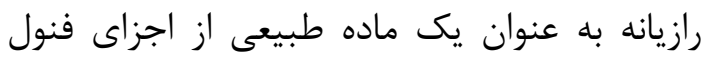

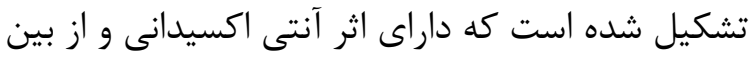

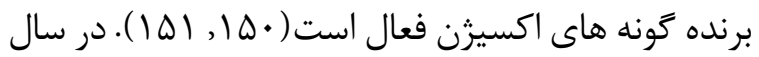

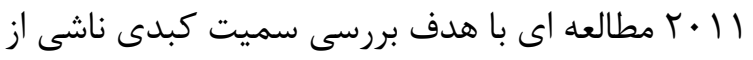
CPF

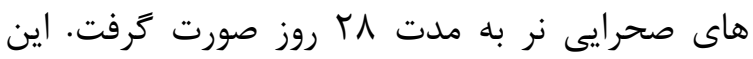

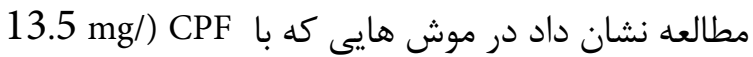

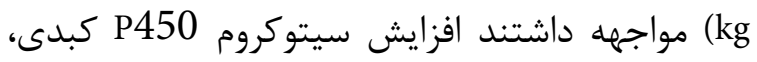

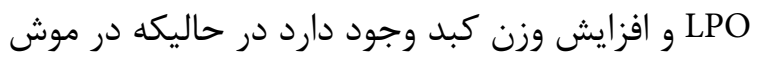

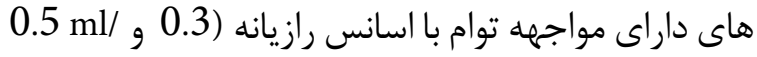

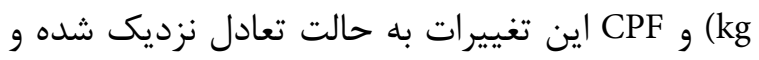

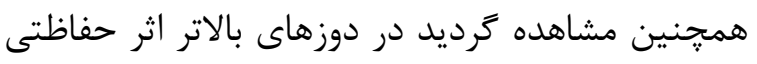

اسانس رازيانه بيشتر است(ع (1) (1).

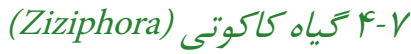

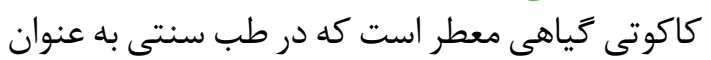

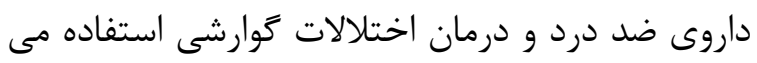

(Opuntia ficus indica)

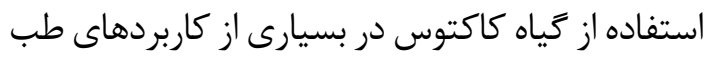

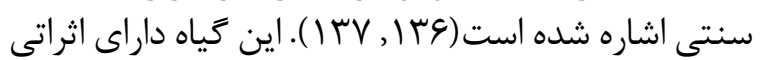

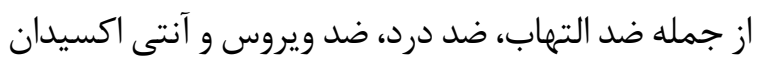

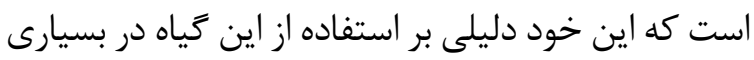

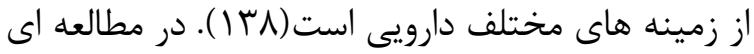

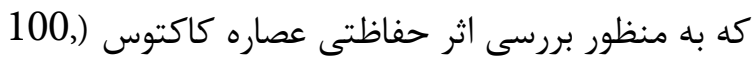

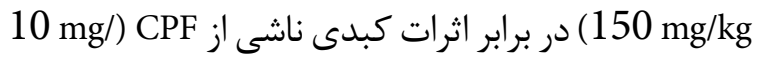

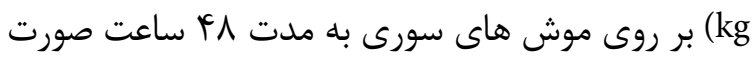

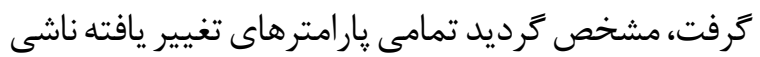
از مواجها با CPF از جمله

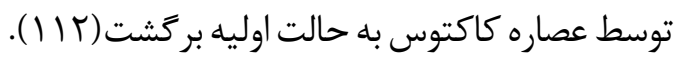

(Curcumin) كوركومين كور كومين داراى طيف گسترده اي از خواص دارين إرويى

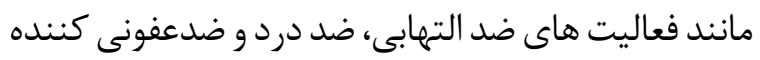

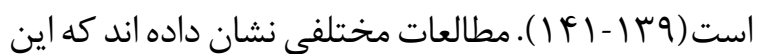

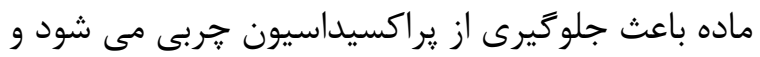

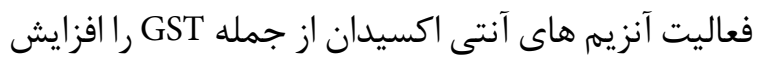

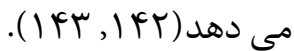

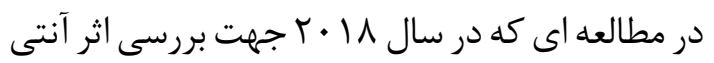
اكسيدانى كور كومين در برابر سميت كبدى در ناشى از از

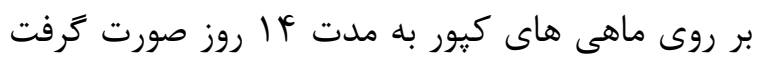

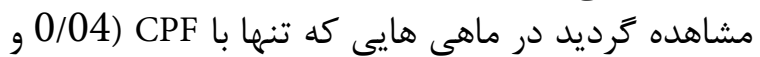

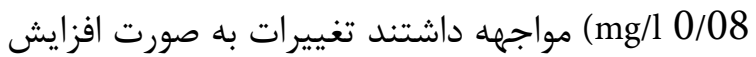

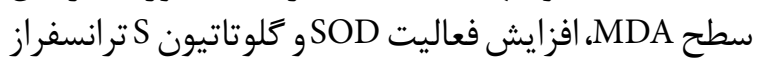
همجنين كاهش سطح CAT و GPx وجود داشت اما در

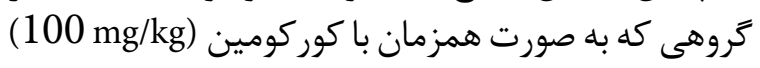

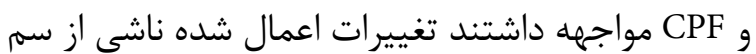

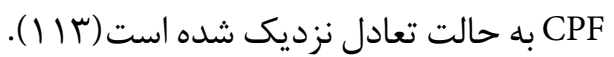

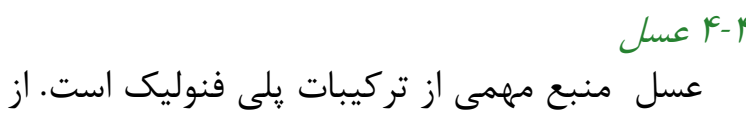

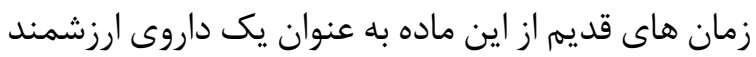

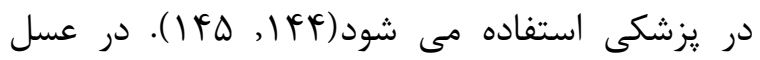

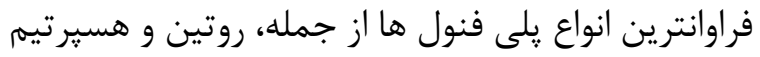

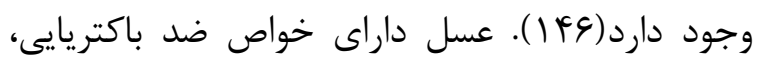

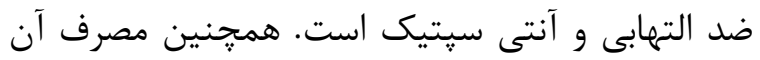
براى افراد مبتلا به بيمارى هاى جشمى آني، قلبى -عروقى آنى

1 amino transferase

2 aspartate amino transferase

3 phosphatase alkaline

4 lactate dehydrogenase 
آسيارتات و آلانين آمينوترانسفراز به طور جشمخيرى

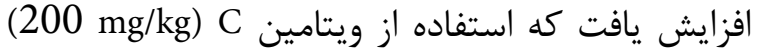

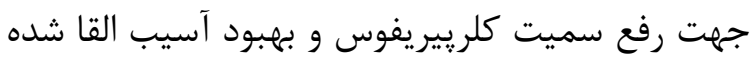

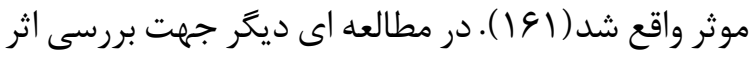

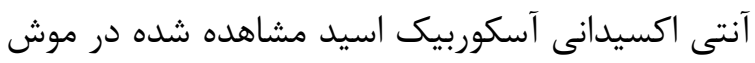

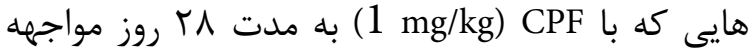

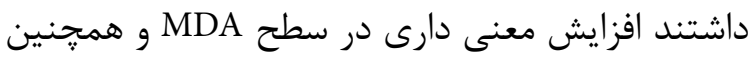

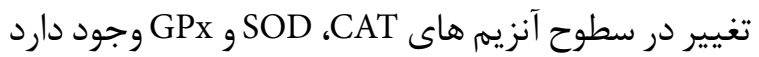

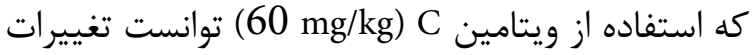

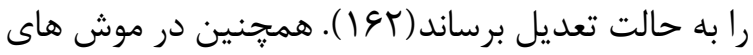

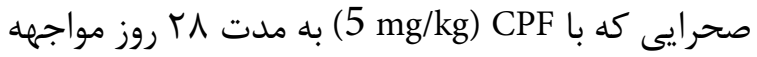

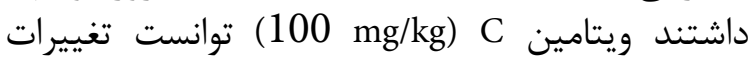

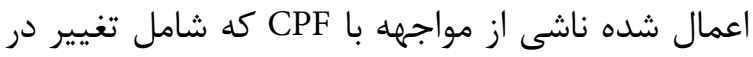

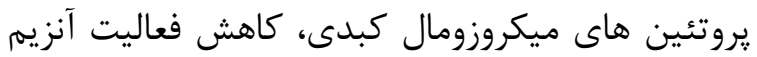

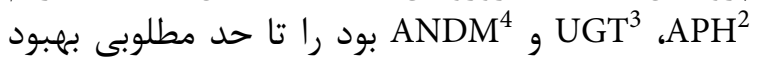

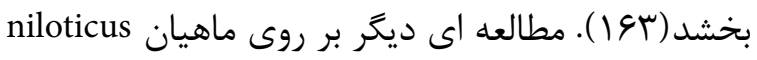

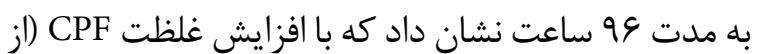

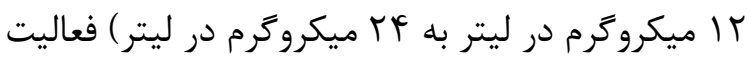

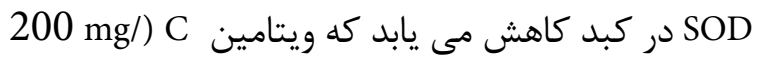

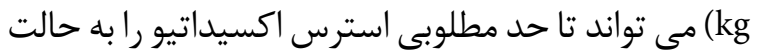

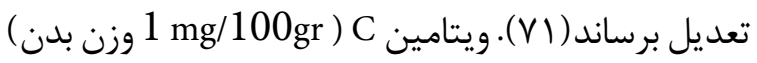

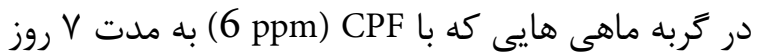

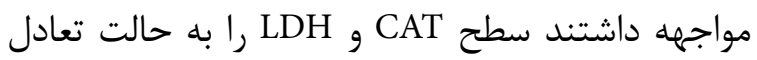

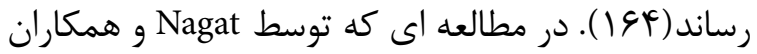

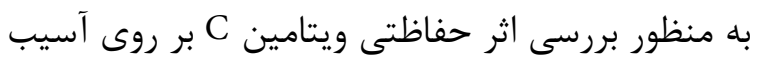

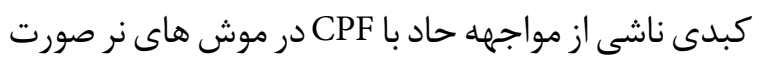

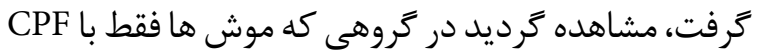

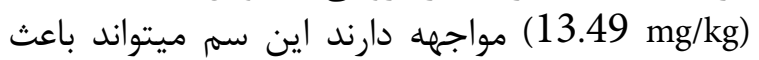

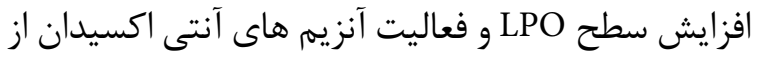

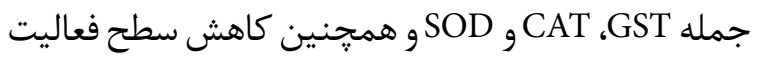

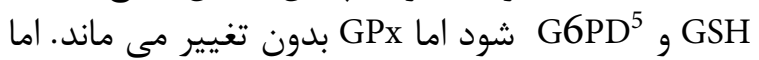

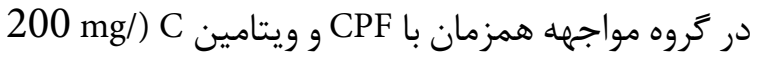

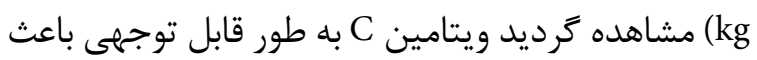

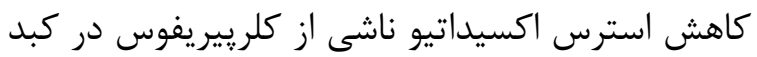

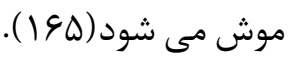

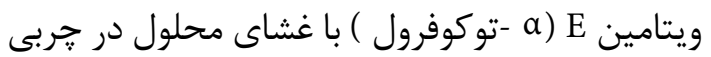

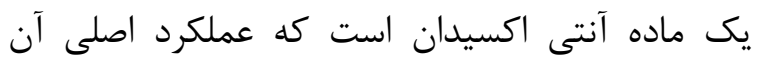

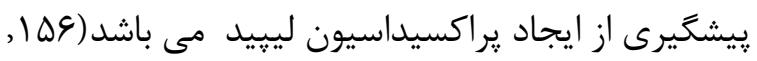

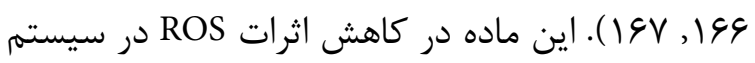

2 aniline p-hydroxylase

3 uridine diphosphate glucuronosyltransferase

4 aminopyrine $\mathrm{N}$-demethylase

5 glucose-6-phosphate dehydrogenase
شود( S ( ) ). همجنين مطالعات بيولوزيكى نشان داده اند

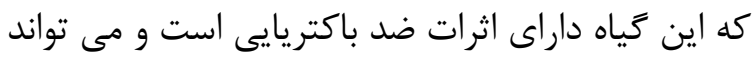

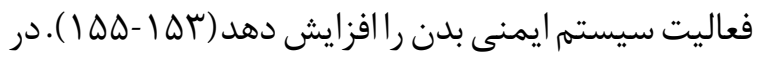

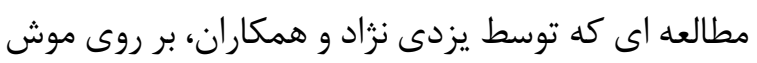

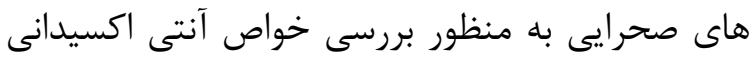

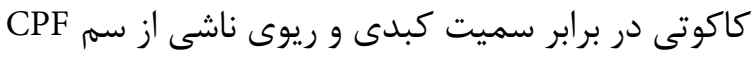

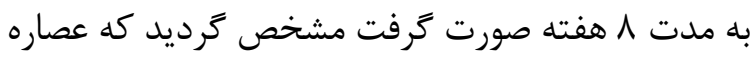

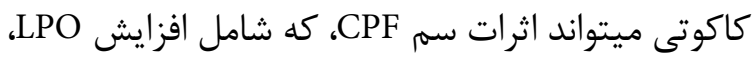

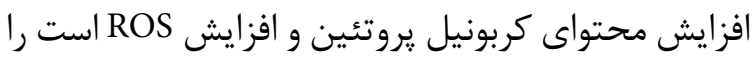
به صورت جشمخيرى كاهش دهد (1)V (1).

ه- مواد غيرفنولى جهت حفاظت در برابر سميت كبدى

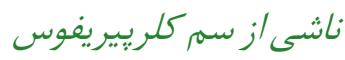

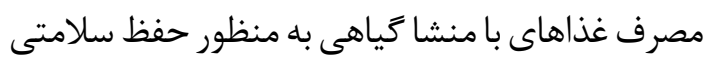

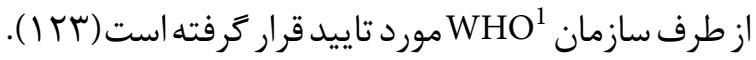

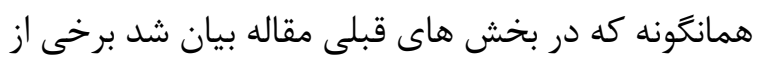

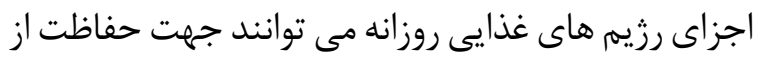

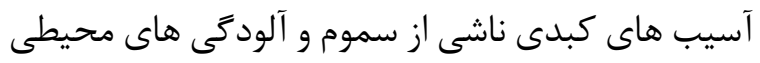

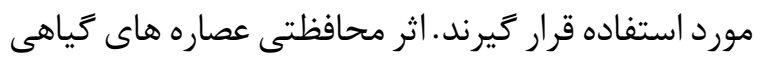

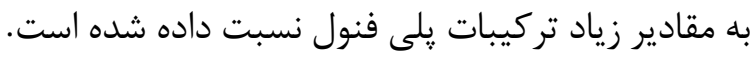

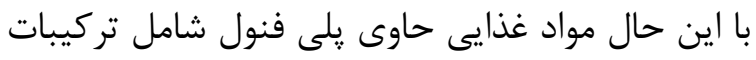

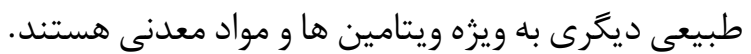

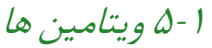

ويتامين ها از جمله ويتامين Cو C مواد غذايى ضرورى

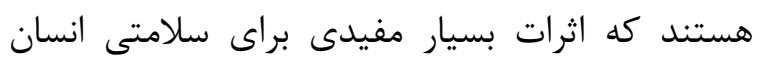

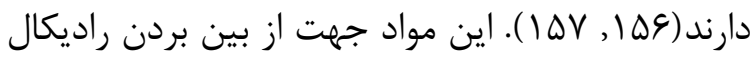

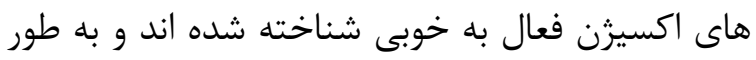

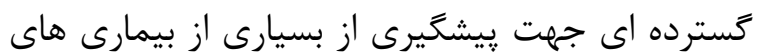

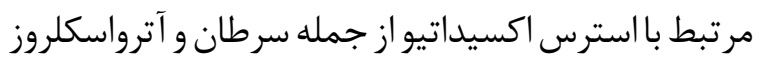

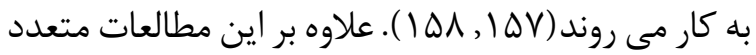

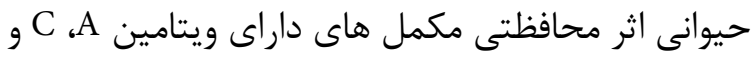
E

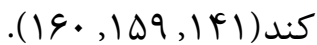

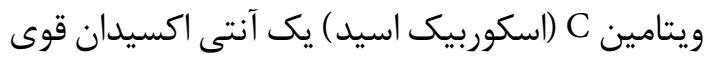

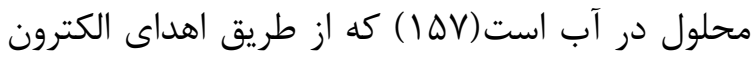

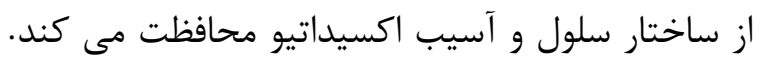

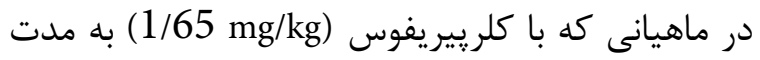

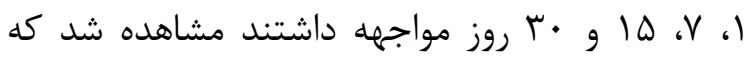

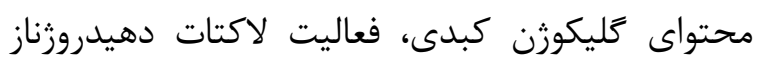
و آمينوترانسفراز كاهش و فعاليت كايت مالات دهيدروزناز ، دهيدروناز

1 World Health Organization

Iran Occupational Health. 2021 (01 May);18: 9. 
(IV9 )، همجنين در فرايندهاى فيزيولوزيكى از قبيل رشد (IVY)

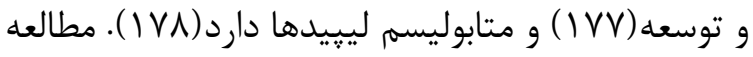

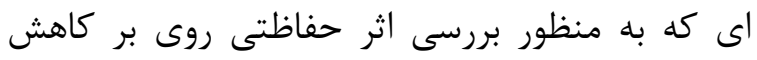

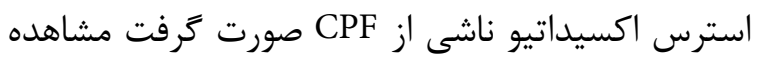

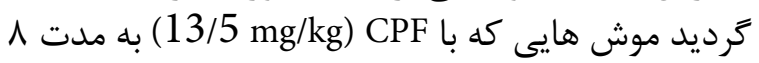

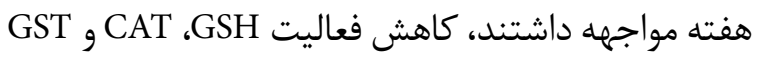

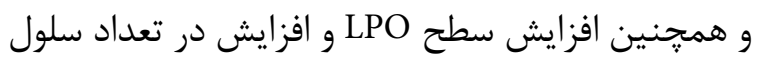
هاى دوقلو و انقباض سينوس ها ها وجود دارد كه مصرف

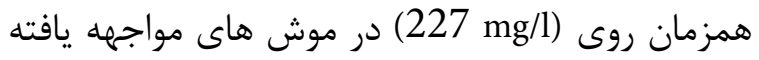

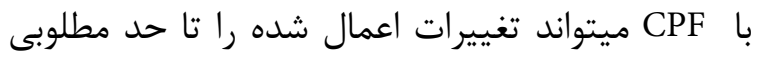

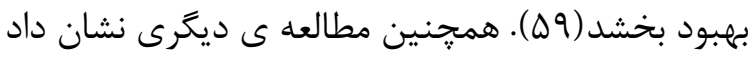

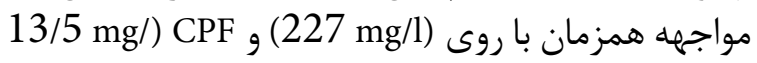

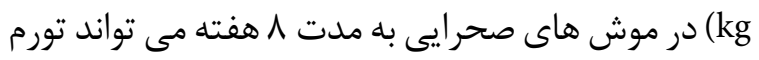

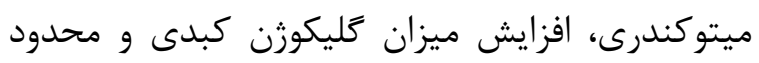

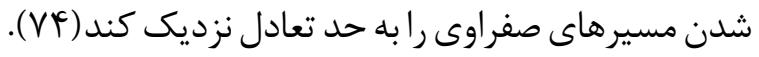

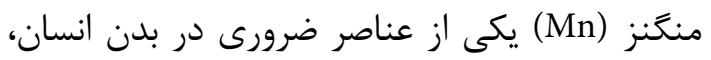

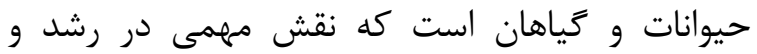

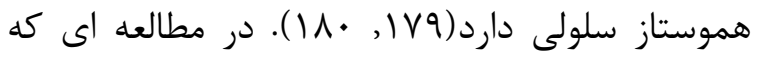

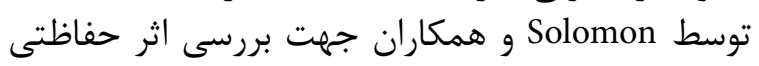

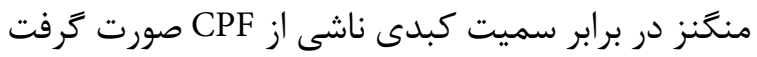

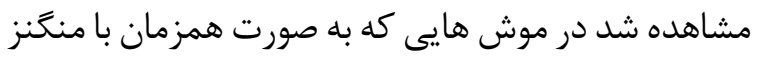

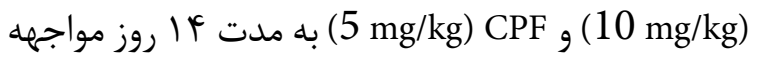

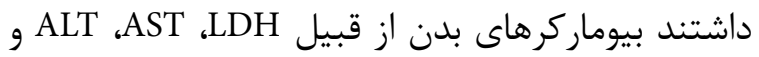

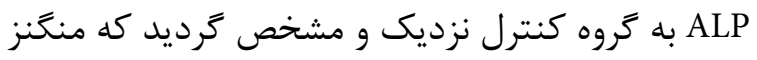

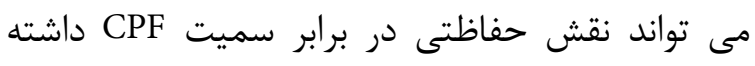

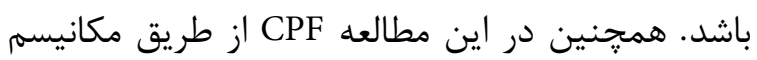

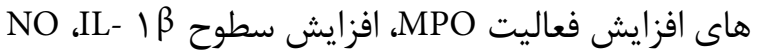

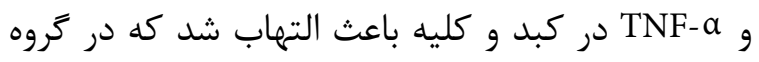

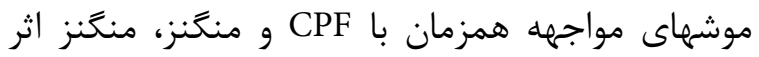

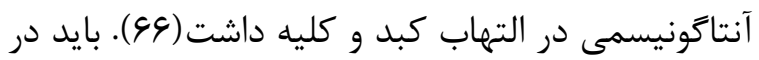

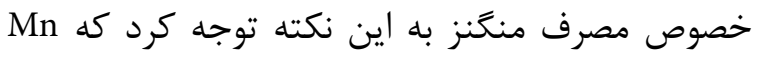

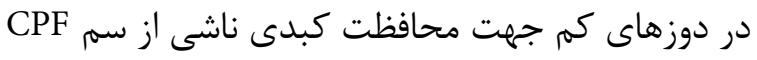

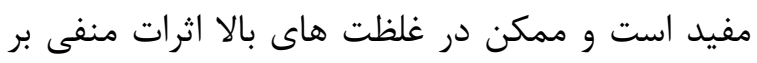

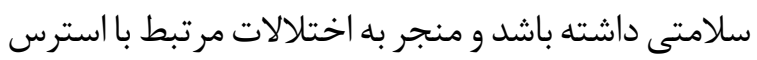

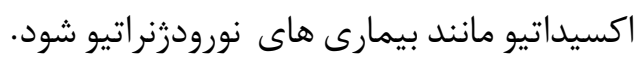

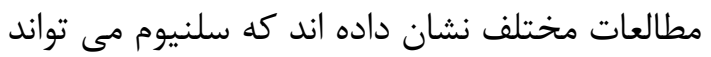

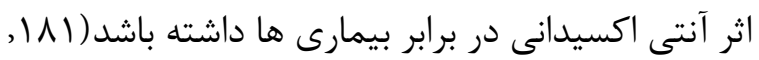

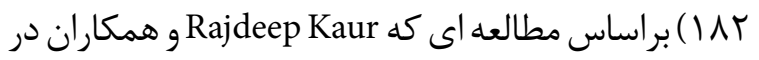

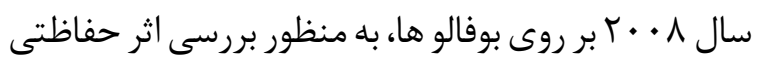

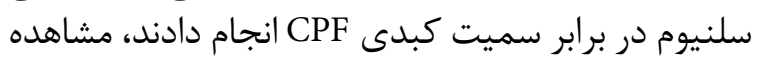

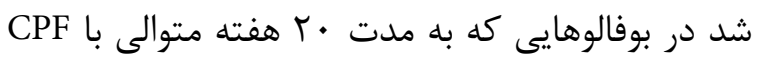

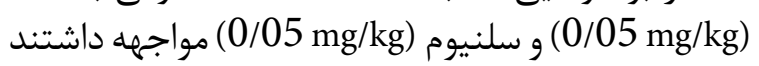

هاى بيولوزيكى و حذف راديكال هاى آزاد موثر است (1 أ ) .

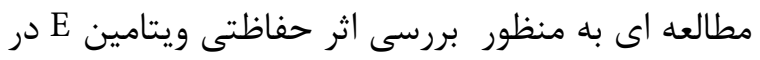

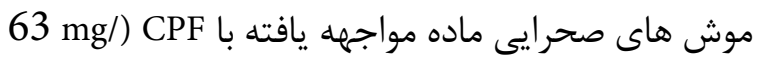

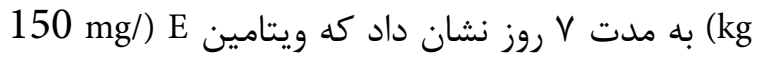

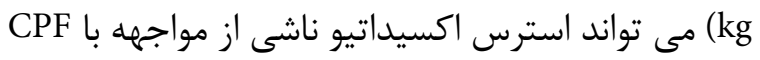

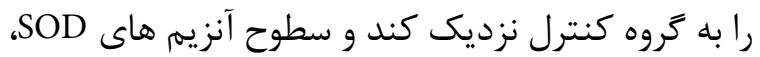

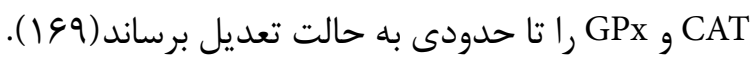

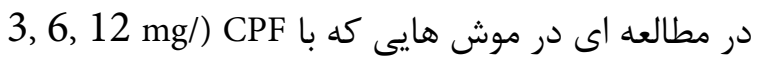

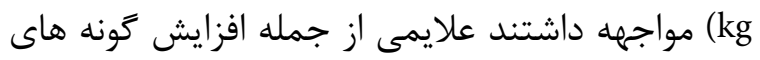

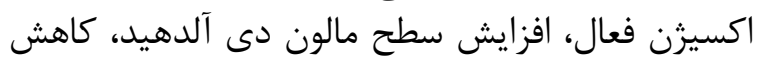

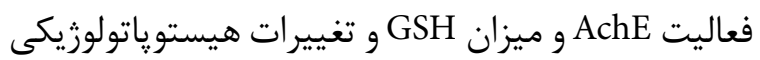

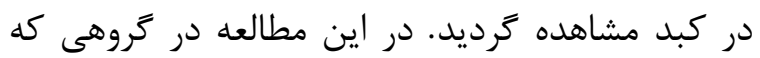

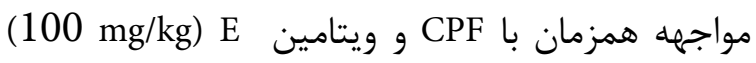

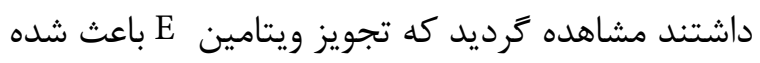

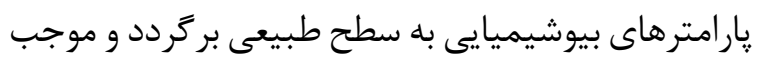

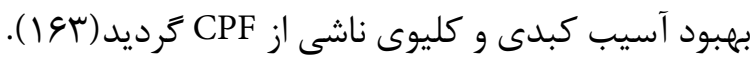

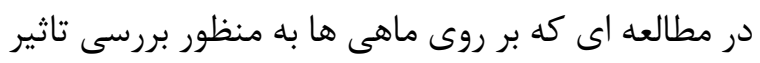

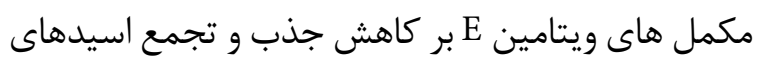

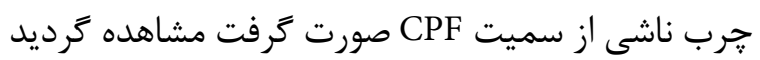

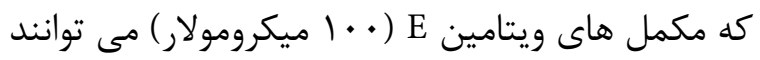

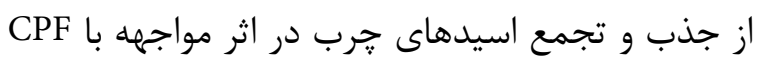

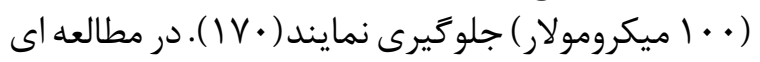

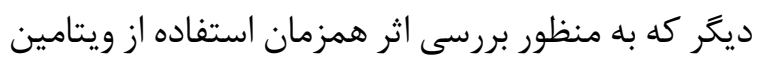

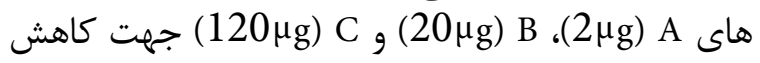

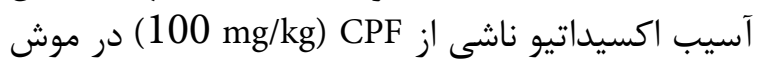

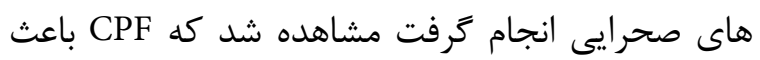

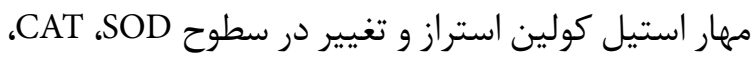
G6PDH و همجنين تجمع 'TBARS اما در موش هايى كه همزمان با سمر و مواد آنتى اكسيدان

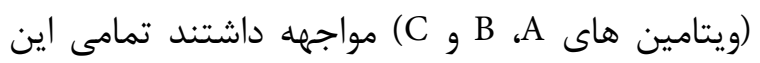

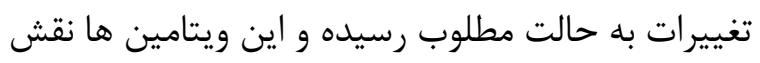

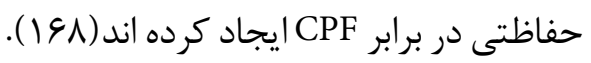

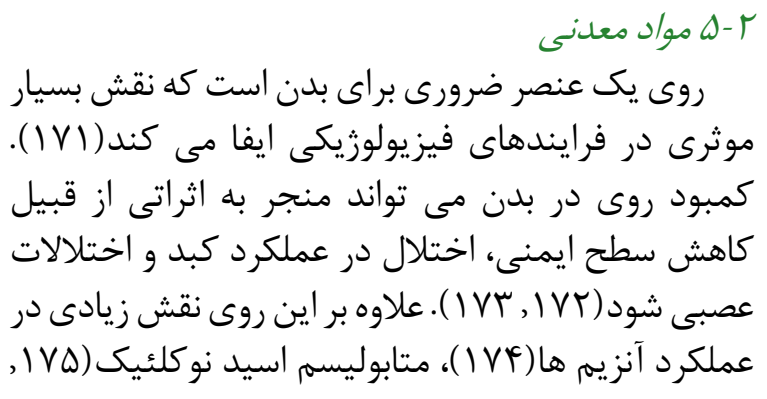

1 Glucose-6-phosphate dehydrogenase

2 thiobarbituric acid reactive substances 
كبد يك اندام اصلى در متابوليسم سموم است، بررسى راه هاى جلوكيرى از ايجاد استرس اكسيداتيو در ائن اين اندام

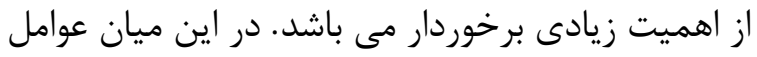

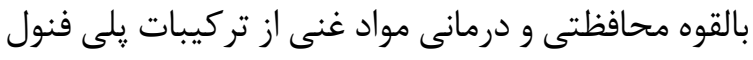

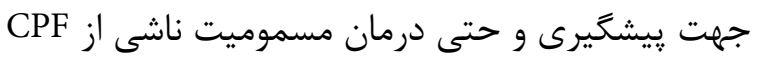

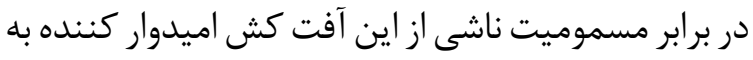

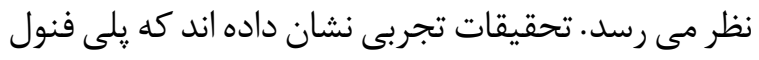

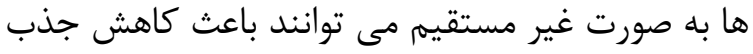

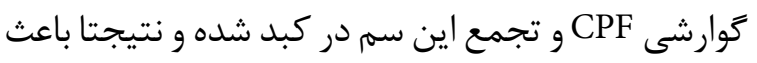

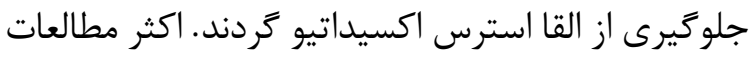

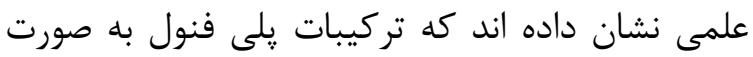

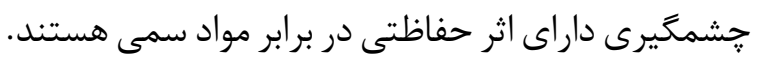

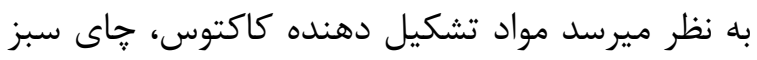

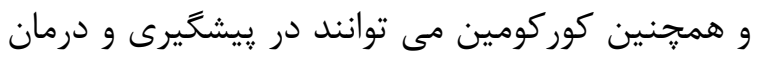

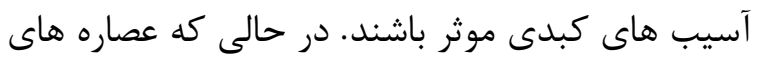

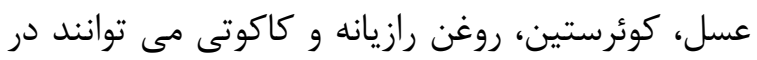
درمان آسيب هاى ناشى از

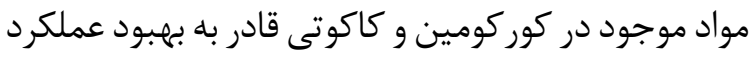

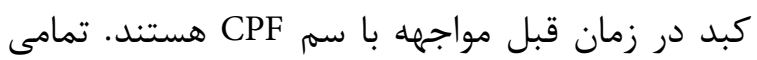

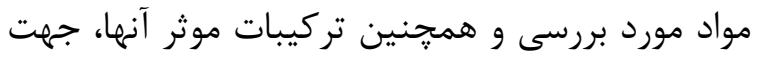

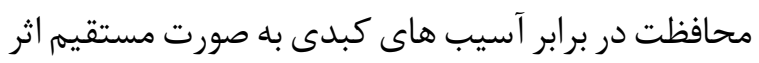

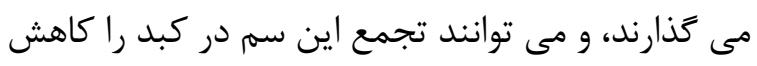

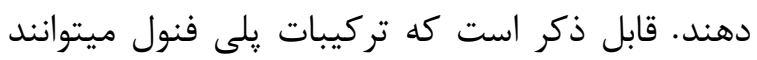

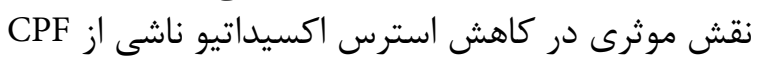

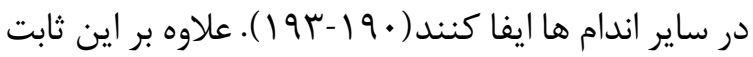

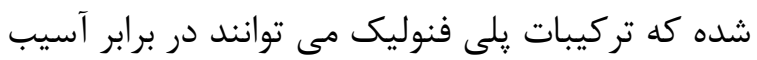

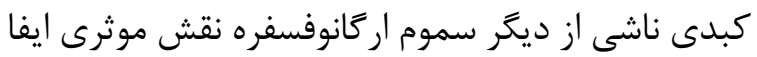

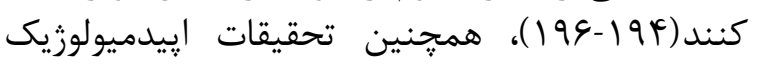

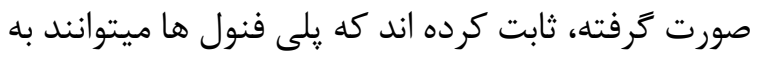

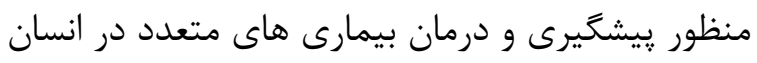

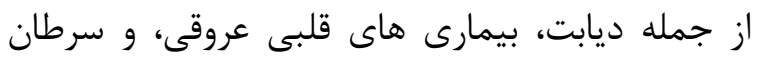

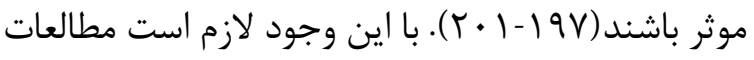

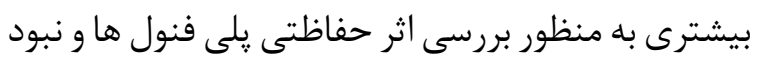
اثرات جانبى در برابر سم CPF در انسان انجام كردد.

\section{References}

1. Pourbabaki R, Khadem M, Samiei S, Hasanpour H, Shahtaheri SJ. The protective effect of rosemary in mitigating oxidative stress induced by Chlorpyrifos in rat kidney. Journal of Health and Safety at Work. 2020;10(2):24-9.

2. Gunnell D, Eddleston M, Phillips MR, Konradsen F. The global distribution of fatal pesticide self-poisoning:
سلنيوم تاثير مثبتى بر روى يتانسيل آنتى اكسيدانى كبد (SODT (آنيد

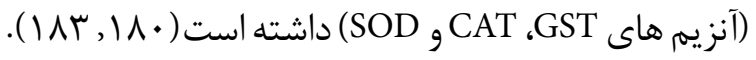

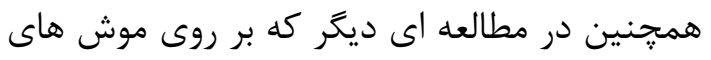

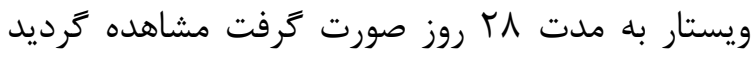

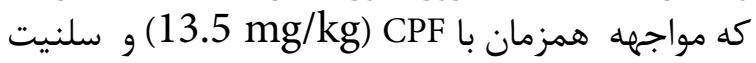

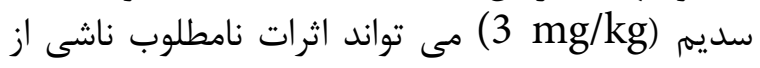

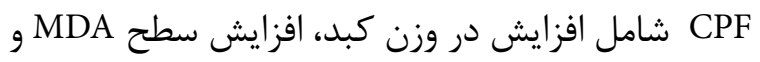

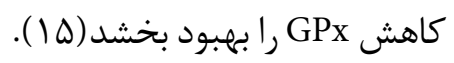

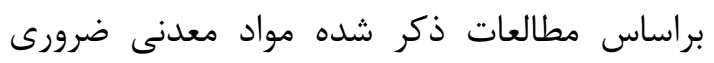

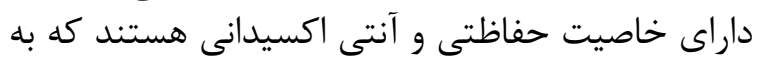

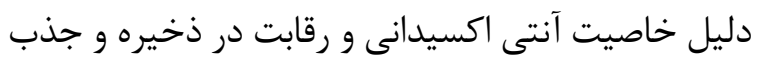

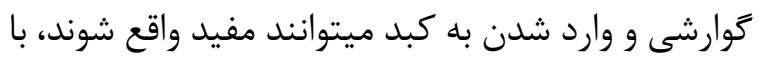

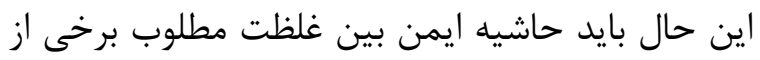

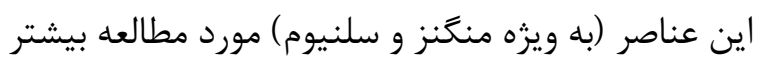
قرار زيرد.

" - ه ديَر تركيبات سرشار از مواد يلى فنول

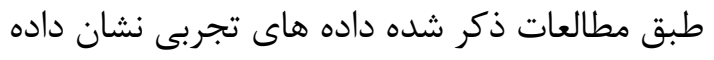

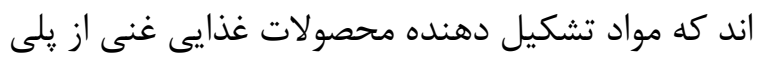

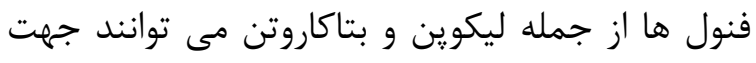

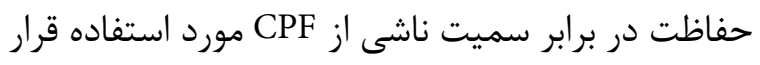
كيرند (شكل ع).

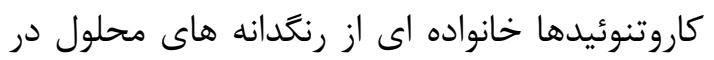

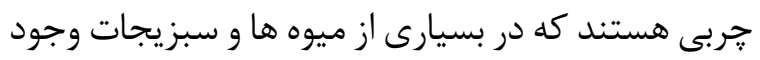

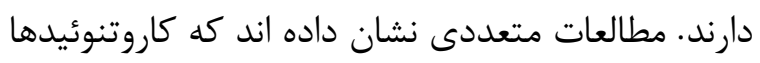
مى توانند جهت كاهش استرس اكسيداتيو استفاده شوند.

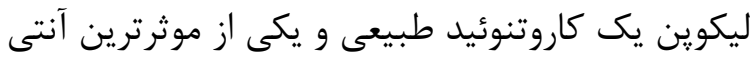

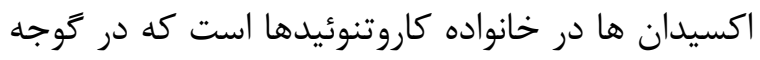
فرنكى وجود دارد(1)

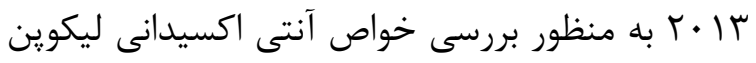

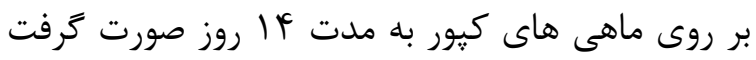

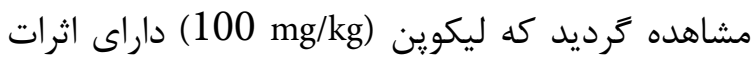

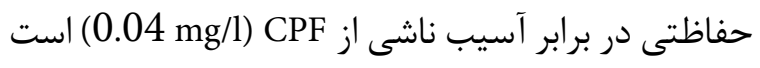

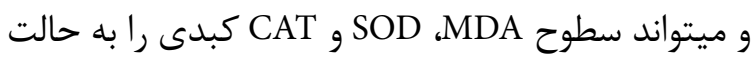

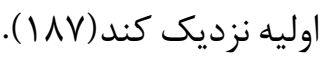

$$
\text { نتيجه تَيرى }
$$

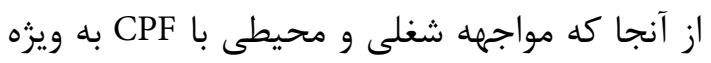

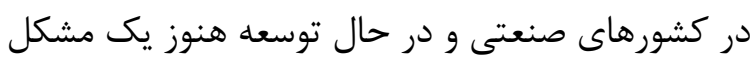

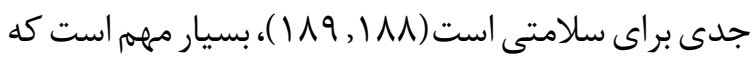

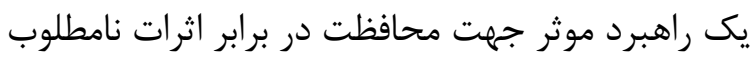

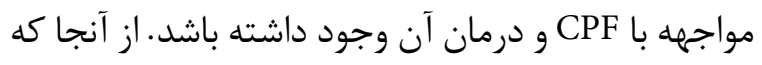


and central $\mathrm{U}$ nited $\mathrm{S}$ tates: patterns and drivers. Global Change Biology. 2011;17(11):3312-26.

14. Jahani AJIJoF, Research P. Modeling of forest canopy density confusion in environmental assessment using artificial neural network. 2016;24(2).

15. Heikal TM, El-Sherbiny M, Hassan SA, Arafa A, Ghanem HZJIJPPS. Antioxidant effect of selenium on hepatotoxicity induced by chlorpyrifos in male rats. 2012;4(4):603-9.

16. Rai DK, Sharma BJMb. Carbofuran-induced oxidative stress in mammalian brain. 2007;37(1):66.

17. Heikal T, Soliman MJJEST. Effect of fish oil supplementation on brain DNA damage and hepatic oxidant/antioxidant status in dimethoate-treated rats. 2010;42:1-9.

18. Heikal T, Ghanem H, Soliman MJBSB. Protective effect of green tea extracts against dimethoate induced DNA damage and oxidant/antioxidant status in male rats. 2011;3(1):1-11.

19. Celik I, Yilmaz Z, Turkoglu VJETAIJ. Hematotoxic and hepatotoxic effects of dichlorvos at sublethal dosages in rats. 2009;24(2):128-32.

20. Halliwell B, Gutteridge JM. Free radicals in biology and medicine: Oxford University Press, USA; 2015.

21. Kalender S, Uzun FG, Durak D, Demir F, Kalender YJF, toxicology c. Malathion-induced hepatotoxicity in rats: the effects of vitamins C and E. 2010;48(2):633-8.

22. Kalantary S, Jahani A, Pourbabaki R, Beigzadeh Z. Application of ANN modeling techniques in the prediction of the diameter of PCL/gelatin nanofibers in environmental and medical studies. RSC advances. 2019;9(43):24858-74.

23. Vuillaume MJMRRiGT. Reduced oxygen species, mutation, induction and cancer initiation. 1987;186(1):43-72.

24. Guicciardi M, Malhi H, Mott J, Gores G. Apoptosis and necrosis in the liver. Compr Physiol 3: 977-1010. John Wiley \& Sons Inc PubMed Google Scholar; 2013.

25. Kalantary S, Pourbabaki R, Jahani A, Sadeghi Yarandi M, Samiei S, Jahani R. Development of a decision support system tool to predict the pulmonary function using artificial neural network approach. Concurrency and Computation: Practice and Experience.e6258.

26. Björnsson ES, Hoofnagle JH. Categorization of drugs implicated in causing liver injury: critical assessment based on published case reports. Hepatology. 2016;63(2):590-603.

27. Stine JG, Chalasani NPJCild. Drug hepatotoxicity: environmental factors. 2017;21(1):103-13.

28. Malaguarnera G, Cataudella E, Giordano M, Nunnari G, Chisari G, Malaguarnera MJWjogW. Toxic hepatitis in occupational exposure to solvents. 2012;18(22):2756. systematic review. BMC public health. 2007;7(1):357.

3. Bakand S, Dehghani Y, Gohari M, Mosadegh M, Mirmohammadi S. Exposure assessment of greenhouse workers with anti-cholinesterase pesticides by biological monitoring. 2012.

4. Soltaninejad K, Abdollahi M. Current opinion on the science of organophosphate pesticides and toxic stress: a systematic review. Medical Science Monitor. 2009;15(3):RA75-RA90.

5. Karami-Mohajeri S, Ahmadipour A, Rahimi H-R, Abdollahi M. Adverse effects of organophosphorus pesticides on the liver: a brief summary of four decades of research. Archives of Industrial Hygiene and Toxicology. 2017;68(4):261-75.

6. Kalantary S, Golbabaei F, Latifi M, Shokrgozar MA, Yaseri MJJon, nanotechnology. Feasibility of Using Vitamin E-Loaded Poly ( $\varepsilon$-caprolactone)/Gelatin Nanofibrous Mat to Prevent Oxidative Stress in Skin. 2020;20(6):3554-62.

7. Sadeghi-Yarandi M, Golbabaei F, Karimi A. Evaluation of pulmonary function and respiratory symptoms among workers exposed to 1, 3-Butadiene in a petrochemical industry in Iran. Archives of environmental \& occupational health. 2020;75(8):48390.

8. Amiri H, Nabizadeh R, Martinez SS, Shahtaheri SJ, Yaghmaeian K, Badiei A, et al. Response surface methodology modeling to improve degradation of Chlorpyrifos in agriculture runoff using $\mathrm{TiO} 2$ solar photocatalytic in a raceway pond reactor. 2018;147:91925.

9. Lee I, Eriksson P, Fredriksson A, Buratovic S, Viberg H. Developmental neurotoxic effects of two pesticides: Behavior and biomolecular studies on chlorpyrifos and carbaryl. Toxicology and applied pharmacology. 2015;288(3):429-38.

10. Yanai J, Brick-Turin Y, Dotan S, Langford R, Pinkas A, Slotkin TA. A mechanism-based complementary screening approach for the amelioration and reversal of neurobehavioral teratogenicity. Neurotoxicology and teratology. 2010;32(1):109-13.

11. Alaa-Eldin EA, El-Shafei DA, Abouhashem NS. Individual and combined effect of chlorpyrifos and cypermethrin on reproductive system of adult male albino rats. Environmental Science and Pollution Research. 2017;24(2):1532-43.

12. Ventura C, Zappia C, Lasagna M, Pavicic W, Richard S, Bolzan A, et al. Effects of the pesticide chlorpyrifos on breast cancer disease. Implication of epigenetic mechanisms. The Journal of steroid biochemistry and molecular biology. 2018.

13. Dietze MC, Moorcroft PR. Tree mortality in the eastern 
استفاده از تركيبات سرشار از مواد آنتى اكسيدان ....

World Health Organization; 2010.

42. Patlolla AK, Barnes C, Yedjou C, Velma V, Tchounwou PB. Oxidative stress, DNA damage, and antioxidant enzyme activity induced by hexavalent chromium in Sprague-Dawley rats. Environmental Toxicology: An International Journal. 2009;24(1):66-73.

43. Sánchez-Valle V, C Chavez-Tapia N, Uribe M, MéndezSánchez N. Role of oxidative stress and molecular changes in liver fibrosis: a review. Current medicinal chemistry. 2012;19(28):4850-60.

44. Sakaguchi S, Takahashi S, Sasaki T, Kumagai T, Nagata KJDm, pharmacokinetics. Progression of alcoholic or non-alcoholic steatohepatitis; common metabolic aspects of innate immune system and oxidative stress. 2010:1011300126-.

45. Cichoż-Lach H, Michalak AJWjogW. Oxidative stress as a crucial factor in liver diseases. 2014;20(25):8082.

46. Wu D, Cederbaum AI, editors. Oxidative stress and alcoholic liver disease. Seminars in liver disease; 2009: (c) Thieme Medical Publishers.

47. Feng Y, Wang N, Ye X, Li H, Feng Y, Cheung F, et al. Hepatoprotective effect and its possible mechanism of Coptidis rhizoma aqueous extract on carbon tetrachloride-induced chronic liver hepatotoxicity in rats. 2011;138(3):683-90.

48. Singal AK, Jampana SC, Weinman SAJLI. Antioxidants as therapeutic agents for liver disease. 2011;31(10):143248.

49. Li A-N, Li S, Zhang Y-J, Xu X-R, Chen Y-M, Li $\mathrm{H}-\mathrm{BJN}$. Resources and biological activities of natural polyphenols. 2014;6(12):6020-47.

50. Brent J, Wallace K, Burkhart K, Phillips S, Donovan J. Critical care toxicology, diagnosis and management of the critically poisoned patient. Mosby. Inc; 2005.

51. . !!! INVALID CITATION !!! (31-33).

52. Wang L, Liu Z, Zhang J, Wu Y, Sun HJEr. Chlorpyrifos exposure in farmers and urban adults: Metabolic characteristic, exposure estimation, and potential effect of oxidative damage. 2016;149:164-70.

53. Robertson V. The assessment of potential insecticidal plants for local use in rural highland Ecuador: University of British Columbia; 2010.

54. Goel A, Chauhan D, Dhawan D. Protective effects of zinc in chlorpyrifos induced hepatotoxicity. Biological trace element research. 2000;74(2):171.

55. Hodgson E, Rose RL. Metabolic interactions of agrochemicals in humans. Pest Management Science: formerly Pesticide Science. 2008;64(6):617-21.

56. Mikhail T, Aggour N, Awadallah R, Boulos M, El-Dessoukey E, Karima A. Acute toxicity of organophosphorus and organochlorine insecticides in laboratory animals. Zeitschrift für
29. Authority EFS. Cadmium dietary exposure in the European population. EFSA Journal. 2012;10(1):2551.

30. Fagerberg B, Barregard L, Sallsten G, Forsgard N, Östling G, Persson M, et al. Cadmium exposure and atherosclerotic carotid plaques-Results from the Malmö diet and Cancer study. Environmental research. 2015; 136:67-74.

31. Larsen JC. Scientific Opinion of the Panel on Contaminants in the Food Chain on a request from the European Commission on Polycyclic Aromatic Hydrocarbons in Food: Question N EFSA-Q-2007-136. 2008.

32. Nogawa K, Sakurai M, Ishizaki M, Kido T, Nakagawa $\mathrm{H}$, Suwazono $\mathrm{Y}$. Threshold limit values of the cadmium concentration in rice in the development of itai-itai disease using benchmark dose analysis. Journal of applied toxicology. 2017;37(8):962-6.

33. Organization WH. International travel and health: situation as on 1 January 2010: World Health Organization; 2010.

34. Akbarifard S, Sharifi MR, Qaderi KJDiB. Data on Optimization of the Karun-4 Hydropower Reservoir Operation Using Evolutionary Algorithms. 2020:105048.

35. Mezynska M, Brzoska MM. Environmental exposure to cadmium-A risk for health of the general population in industrialized countries and preventive strategies. Environmental Science and Pollution Research. 2018;25(4):3211-32.

36. Organization WH. Exposure to arsenic: a major public health concern. Geneva: Public Health and Environment. 2010;1.

37. JAHANI A, MAKHDOUM F, FEGHHI J, OMID M. ENVIRONMENTAL DECISION SUPPORT SYSTEMS (EDSSS): THE STUDY OF CONCEPTS, DEVELOPMENTS AND CHALLENGES FROM PAST TO PRESENT. 2016.

38. Hyder O, Chung M, Cosgrove D, Herman JM, Li Z, Firoozmand A, et al. Cadmium exposure and liver disease among US adults. Journal of Gastrointestinal Surgery. 2013;17(7):1265-73.

39. Satarug S, Vesey DA, Gobe GC. Health risk assessment of dietary cadmium intake: do current guidelines indicate how much is safe? Environmental health perspectives. 2017;125(3):284.

40. Yasser E-N, Lubbad R. Acute and single repeated dose effects of low concentrations of chlorpyrifos, diuron, and their combination on chicken. Environmental Science and Pollution Research. 2018;25(11):10837-47.

41. Meeting JFWECoFA, Organization WH. Evaluation of Certain Food Additives: Seventy-first Report of the Joint FAO/WHO Expert Committee on Food Additives: 
70. Mehta A, Verma RS, Srivastava N. Chlorpyrifos-induced DNA damage in rat liver and brain. Environmental and molecular mutagenesis. 2008;49(6):426-33.

71. Özkan F, Gündüz SG, Berköz M, Hunt AÖ, Yalın S. The protective role of ascorbic acid (vitamin C) against chlorpyrifos-induced oxidative stress in Oreochromis niloticus. Fish physiology and biochemistry. 2012;38(3):635-43.

72. Fereshteh Esfandyar, Farid Firouzbakhsh, Hossein Rahmani, Jani-Khalili K. The effects of Sublethal Concentrations of Chlorpyrifos on the Liver Enzyme Activities and Oxidative Stress Markers in Common Carp (Cyprinus carpio). 2016;69(3):229-307.

73. Xing H, Li S, Wang Z, Gao X, Xu S, Wang X. Oxidative stress response and histopathological changes due to atrazine and chlorpyrifos exposure in common carp. Pesticide biochemistry and physiology. 2012;103(1):7480.

74. Goel A, Dhawan DKJBter. Zinc supplementation prevents liver injury in chlorpyrifos-treated rats. 2001;82(1-3):185.

75. Tripathi S, Srivastav AK. Liver profile of rats after long-term ingestion of different doses of chlorpyrifos. Pesticide biochemistry and physiology. 2010;97(1):605.

76. Tuzmen N, Candan N, Kaya E, Demiryas N. Biochemical effects of chlorpyrifos and deltamethrin on altered antioxidative defense mechanisms and lipid peroxidation in rat liver. Cell Biochemistry and Function. 2008;26(1):119-24.

77. Stenersen J. Chemical pesticides mode of action and toxicology: CRC press; 2004.

78. Ghavidel F, Shahtaheri SJ, Torabbeigi M, Rahimi Froushani AJACL. Microwave assisted head space solid phase microextraction for analysis of butachlor and chlorpyrifos pesticides in urine. 2014;4(4):224-31.

79. Rahiminezhad M, Shahtaheri SJ, Ganjali MR, Koohpaei A-R, Forushani AR, Golbabaei FJIjooh. Synthesis of molecularly imprinted polymer as a solid phase sorbent for pesticide Dursban. 2010:51-6.

80. Solomon KR, Williams WM, Mackay D, Purdy J, Giddings JM, Giesy JP. Properties and uses of chlorpyrifos in the United States. Ecological Risk Assessment for Chlorpyrifos in Terrestrial and Aquatic Systems in the United States: Springer, Cham; 2014. p. 13-34.

81. Poet TS, Timchalk C, Hotchkiss JA, Bartels MJJX. Chlorpyrifos PBPK/PD model for multiple routes of exposure. 2014;44(10):868-81.

82. Chambers JE, Levi PE. Organophosphates: chemistry, fate, and effects: Academic Press San Diego; 1992.

83. . !!! INVALID CITATION !!! (65-67).
Ernährungswissenschaft. 1979;18(4):258-68.

57. Mansour SA, Mossa A-THJT, health i. Adverse effects of exposure to low doses of chlorpyrifos in lactating rats. 2011;27(3):213-24.

58. Chambers JE, Chambers HWJJobt. Oxidative desulfuration of chlorpyrifos, chlorpyrifos-methyl, and leptophos by rat brain and liver. 1989;4(3):201-3.

59. Goel A, Dani V, Dhawan D. Protective effects of zinc on lipid peroxidation, antioxidant enzymes and hepatic histoarchitecture in chlorpyrifos-induced toxicity. Chemico-Biological Interactions. 2005;156(2-3):13140.

60. Ezzi L, Salah IB, Haouas Z, Sakly A, Grissa I, Chakroun $S$, et al. Histopathological and genotoxic effects of chlorpyrifos in rats. Environmental Science and Pollution Research. 2016;23(5):4859-67.

61. Tripathi S, Srivastav A. Toxicology and Health. 2010.

62. Topal A, Atamanalp M, Oruç E, Demir Y, Beydemir Ş, Işı $\mathrm{A}$. In vivo changes in carbonic anhydrase activity and histopathology of gill and liver tissues after acute exposure to chlorpyrifos in rainbow trout. Archives of Industrial Hygiene and Toxicology. 2014;65(4):377-85.

63. Ma J, Liu Y, Niu D, Li X. Effects of chlorpyrifos on the transcription of CYP3A cDNA, activity of acetylcholinesterase, and oxidative stress response of goldfish (Carassius auratus). Environmental toxicology. 2015;30(4):422-9.

64. Abolaji A, Awogbindin I, Adedara I, Farombi E. Insecticide chlorpyrifos and fungicide carbendazim, common food contaminants mixture, induce hepatic, renal, and splenic oxidative damage in female rats. Human \& experimental toxicology. 2017;36(5):483-93.

65. Zahran E, Risha E, Awadin W, Palić D. Acute exposure to chlorpyrifos induces reversible changes in health parameters of Nile tilapia (Oreochromis niloticus). Aquatic Toxicology. 2018;197:47-59.

66. Owumi SE, Dim UJJTR. Manganese suppresses oxidative stress, inflammation and caspase- 3 activation in rats exposed to chlorpyrifos. 2019;6:202-9.

67. Khan SM. Protective effect of black tea extract on the levels of lipid peroxidation and antioxidant enzymes in liver of mice with pesticide-induced liver injury. Cell Biochemistry and Function: Cellular biochemistry and its modulation by active agents or disease. 2006;24(4):327-32.

68. Wang J, Wang J, Zhu L, Xie H, Shao B, Hou X. The enzyme toxicity and genotoxicity of chlorpyrifos and its toxic metabolite TCP to zebrafish Danio rerio. Ecotoxicology. 2014;23(10):1858-69.

69. Verma RS, Srivastava N. Effect of chlorpyrifos on thiobarbituric acid reactive substances, scavenging enzymes and glutathione in rat tissues. 2003. 
استفاده از تركيبات سرشار از مواد آنتى اكسيدان ....

96. Ravichandran $R$, Rajendran $M$, Devapiriam D. Antioxidant study of quercetin and their metal complex and determination of stability constant by spectrophotometry method. Food chemistry. 2014;146:472-8.

97. Hu R, He Y, Arowolo MA, Wu S, He JJA. Polyphenols as Potential Attenuators of Heat Stress in Poultry Production. 2019;8(3):67.

98. Ni C-X, Gong H, Liu Y, Qi Y, Jiang C-L, Zhang J-P. Green tea consumption and the risk of liver cancer: a meta-analysis. Nutrition and cancer. 2017;69(2):21120.

99. Rasines-Perea Z, Teissedre P-L. Grape polyphenols' effects in human cardiovascular diseases and diabetes. Molecules. 2017;22(1):68.

100. Venancio VP, Cipriano PA, Kim H, Antunes LM, Talcott ST, Mertens-Talcott SU. Cocoplum (Chrysobalanus icaco L.) anthocyanins exert anti-inflammatory activity in human colon cancer and non-malignant colon cells. Food \& function. 2017;8(1):307-14.

101. Bozzetto L, Annuzzi G, Pacini G, Costabile G, Vetrani $\mathrm{C}$, Vitale M, et al. Polyphenol-rich diets improve glucose metabolism in people at high cardiometabolic risk: a controlled randomised intervention trial. Diabetologia. 2015;58(7):1551-60.

102. Henning SM, Wang P, Said JW, Huang M, Grogan T, Elashoff D, et al. Randomized clinical trial of brewed green and black tea in men with prostate cancer prior to prostatectomy. The Prostate. 2015;75(5):550-9.

103. Hokayem M, Blond E, Vidal H, Lambert K, Meugnier E, Feillet-Coudray C, et al. Grape polyphenols prevent fructose-induced oxidative stress and insulin resistance in first-degree relatives of type 2 diabetic patients. Diabetes care. 2013;36(6):1454-61.

104. Tresserra-Rimbau A, Rimm EB, Medina-Remón A, Martínez-González MA, De la Torre R, Corella D, et al. Inverse association between habitual polyphenol intake and incidence of cardiovascular events in the PREDIMED study. Nutrition, Metabolism and Cardiovascular Diseases. 2014;24(6):639-47.

105. Bingül İ, Başaran-Küçükgergin C, Tekkeşin MS, Olgaç V, Doğru-Abbasoğlu S, Uysal M. Effect of blueberry pretreatment on diethylnitrosamine-induced oxidative stress and liver injury in rats. Environmental toxicology and pharmacology. 2013;36(2):529-38.

106. Pang C, Zheng Z, Shi L, Sheng Y, Wei H, Wang Z, et al. Caffeic acid prevents acetaminophen-induced liver injury by activating the Keap1-Nrf2 antioxidative defense system. Free Radical Biology and Medicine. 2016;91:236-46.

107. Choudhury S, Ghosh S, Mukherjee S, Gupta P, Bhattacharya S, Adhikary A, et al. Pomegranate protects
84. Tang J, Cao Y, Rose RL, Brimfield AA, Dai D, Goldstein JA, et al. Metabolism of chlorpyrifos by human cytochrome $\mathrm{P} 450$ isoforms and human, mouse, and rat liver microsomes. 2001;29(9):1201-4.

85. Gorecki L, Korabecny J, Musilek K, Malinak D, Nepovimova E, Dolezal R, et al. SAR study to find optimal cholinesterase reactivator against organophosphorous nerve agents and pesticides. 2016;90(12):2831-59.

86. da Silva LR, Silva B. Natural Bioactive Compounds from Fruits and Vegetables as Health Promoters Part I: Bentham Science Publishers; 2016.

87. ChebabS, Mekircha F, LeghouchiEJB, Pharmacotherapy. Potential protective effect of Pistacia lentiscus oil against chlorpyrifos-induced hormonal changes and oxidative damage in ovaries and thyroid of female rats. 2017;96:1310-6.

88. Alleva R, Manzella N, Gaetani S, Ciarapica V, Bracci $\mathrm{M}$, Caboni MF, et al. Organic honey supplementation reverses pesticide-induced genotoxicity by modulating DNA damage response. 2016;60(10):2243-55.

89. Gultekin F, Delibas N, Yasar S, Kilinc IJAoT. In vivo changes in antioxidant systems and protective role of melatonin and a combination of vitamin $\mathrm{C}$ and vitamin $\mathrm{E}$ on oxidative damage in erythrocytes induced by chlorpyrifos-ethyl in rats. 2001;75(2):88-96.

90. Khalifa FK, Khalil FA, Barakat HA, Hassan MMJAJoB, Sciences A. Protective role of wheat germ and grape seed oils in chlorpyrifos-induced oxidative stress, biochemical and histological alterations in liver of rats. 2011;5(10):54-66.

91. Ambali S, Akanbi D, Igbokwe N, Shittu M, Kawu M, Ayo JJTJots. Evaluation of subchronic chlorpyrifos poisoning on hematological and serum biochemical changes in mice and protective effect of vitamin $\mathrm{C}$. 2007;32(2):111-20.

92. Mansour SA, Mossa A-THJPB, Physiology. Lipid peroxidation and oxidative stress in rat erythrocytes induced by chlorpyrifos and the protective effect of zinc. 2009;93(1):34-9.

93. Quideau S, Deffieux D, Douat-Casassus C, Pouysegu L. Plant polyphenols: chemical properties, biological activities, and synthesis. Angewandte Chemie International Edition. 2011;50(3):586-621.

94. Samiei S, Khadem M, Pourbabaki R, Ghazi-Khansari M, Shahtaheri SJJJoMUoMS. Protective Effect of Salvia officinalis Extract on Deltamethrin-induced Hepatotoxicity in Rats. 2019;29(178):134-40.

95. Borowska S, Brzoska MM, Tomczyk M. Complexation of Bioelements and Toxic Metals by Polyphenolic Compounds-Implications for Health. Current drug targets. 2018;19(14):1612-38. 
Food \& function. 2014;5(4):734-9.

120. Soleimani D, Paknahad Z, Askari G, Iraj B, Feizi A. Effect of garlic powder consumption on body composition in patients with nonalcoholic fatty liver disease: A randomized, double-blind, placebo-controlled trial. Advanced biomedical research. 2016;5.

121. Pezeshki A, Safi S, Feizi A, Askari G, Karami F. The effect of green tea extract supplementation on liver enzymes in patients with nonalcoholic fatty liver disease. International journal of preventive medicine. 2016;7.

122. Heikal TM, Mossa A-TH, Rasoul MAA, MAREI GIK. The ameliorating effects of green tea extract against cyromazine and chlorpyrifos induced liver toxicity in male rats. changes. 2013;5(9).

123. Who J, Organization WH. Diet, nutrition and the prevention of chronic diseases: report of a joint $\mathrm{WH}$. 2003.

124. Grosso G, Stepaniak U, Topor-Mądry R, Szafraniec K, Pająk A. Estimated dietary intake and major food sources of polyphenols in the Polish arm of the HAPIEE study. Nutrition. 2014;30(11-12):1398-403.

125. Taguchi C, Fukushima Y, Kishimoto Y, Suzuki-Sugihara N, Saita E, Takahashi Y, et al. Estimated dietary polyphenol intake and major food and beverage sources among elderly Japanese. Nutrients. 2015;7(12):1026981.

126. Zamora-Ros R, Knaze V, Rothwell JA, Hémon B, Moskal A, Overvad K, et al. Dietary polyphenol intake in Europe: the European Prospective Investigation into Cancer and Nutrition (EPIC) study. European journal of nutrition. 2016;55(4):1359-75.

127. Kelley DS, Adkins YC, Zunino SJ, Woodhouse LR, Bonnel EL, Breksa AP, et al. Citrus limonin glucoside supplementation decreased biomarkers of liver disease and inflammation in overweight human adults. Journal of Functional Foods. 2015;12:271-81.

128. Hoekstra LT, de Graaf W, Nibourg GA, Heger M, Bennink RJ, Stieger B, et al. Physiological and biochemical basis of clinical liver function tests: a review. Annals of surgery. 2013;257(1):27-36.

129. Avsarogullari L, Ikizceli I, Sungur M, gan Sözüer E, Akdur O, Yücei M. Acute amitraz poisoning in adults: clinical features, laboratory findings, and management. Clinical toxicology. 2006;44(1):19-23.

130. Malekirad AA, Faghih M, Mirabdollahi M, Kiani M, Fathi A, Abdollahi M. Neurocognitive, mental health, and glucose disorders in farmers exposed to organophosphorus pesticides. Archives of Industrial Hygiene and Toxicology. 2013;64(1):1-8.

131. Pourhossein M, Shahtaheri S, Mazloumi A, RahimiForoushani A, Helmi-Kohneshahri M, Khani HMJJoac. against arsenic-induced p53-dependent ROS-mediated inflammation and apoptosis in liver cells. The Journal of nutritional biochemistry. 2016;38:25-40.

108. Akbarifard S, Radmanesh FJOE. Predicting sea wave height using Symbiotic Organisms Search (SOS) algorithm. 2018;167:348-56.

109. Uličná O, Vančová O, Kucharská J, Janega P, Waczulíková IJGp, biophysics. Rooibos tea (Aspalathus linearis) ameliorates the CCl4-induced injury to mitochondrial respiratory function and energy production in rat liver. 2019.

110. Yang JH, Choi M-H, Na C-S, Cho SS, Kim JH, Ku SK, et al. Bamboo Stems (Phyllostachys nigra variety henosis) Containing Polyphenol Mixtures Activate Nrf2 and Attenuate Phenylhydrazine-Induced Oxidative Stress and Liver Injury. 2019;11(1):114.

111. Khan SM, Kour GJPB, Physiology. Subacute oral toxicity of chlorpyriphos and protective effect of green tea extract. 2007;89(2):118-23.

112. Ncibi S, Othman MB, Akacha A, Krifi MN, Zourgui LJF, toxicology c. Opuntia ficus indica extract protects against chlorpyrifos-induced damage on mice liver. 2008;46(2):797-802.

113. Yonar MEJE, safety e. Chlorpyrifos-induced biochemical changes in Cyprinus carpio: Ameliorative effect of curcumin. 2018;151:49-54.

114. Tanvir E, Afroz R, Chowdhury MAZ, Khalil MI, Hossain MS, Rahman MA, et al. Honey has a protective effect against chlorpyrifos-induced toxicity on lipid peroxidation, diagnostic markers and hepatic histoarchitecture. 2015;7(5):525-33.

115. Uzun FG, Kalender YJF, toxicology c. Chlorpyrifos induced hepatotoxic and hematologic changes in rats: the role of quercetin and catechin. 2013;55:549-56.

116. Mansour SA, Heikal TM, Refaie AA, Mossa AJGJEST. Antihepatotoxic activity of fennel (Foeniculum vulgare Mill.) essential oil against chlorpyrifos-induced liver injury in rats. 2011;1:10-1.

117. Yazdinezhad A, Abbasian M, Hojjat Hosseini S, Naserzadeh P, Agh-Atabay AH, Hosseini MJJEt. Protective effects of Ziziphora tenuior extract against chlorpyrifos induced liver and lung toxicity in rat: Mechanistic approaches in subchronic study. 2017;32(9):2191-202.

118. Boncheva M, Georgiev G, Shishov V. Effects of Aronia melanocarpa fruit juice in improving medical test results and creating a feeling of health in patients with non-alcoholic fatty liver disease-NAFLD (steatosis). J Gen Med Bulgaria. 2013;2:21-30.

119. Chang H-C, Peng C-H, Yeh D-M, Kao E-S, Wang C-J. Hibiscus sabdariffa extract inhibits obesity and fat accumulation, and improves liver steatosis in humans. 
استفاده از تركيبات سرشار از مواد آنتى اكسيدان ...

acids and flavonoids in honey. 2009;28(7):893-902.

146. Cheng N, Wu L, Zheng J, Cao WJE-BC, Medicine A. Buckwheat honey attenuates carbon tetrachlorideinduced liver and DNA damage in mice. 2015;2015.

147. Renugadevi J, Prabu SMJJoC, Research T. Ameliorative effect of quercetin against cadmium induced toxicity in liver of Wistar rats. 2009;9(1):1665.

148. Sadeghnia HR, Yousefsani BS, Rashidfar M, Boroushaki MT, Asadpour E, Ghorbani AJRf. Protective effect of rutin on hexachlorobutadiene-induced nephrotoxicity. 2013;35(8):1151-5.

149. Ji L-L, Sheng Y-C, Zheng Z-Y, Shi L, Wang Z-TJFRB, Medicine. The involvement of p62-Keap1-Nrf2 antioxidative signaling pathway and JNK in the protection of natural flavonoid quercetin against hepatotoxicity. 2015;85:12-23.

150. Ruberto G, Baratta MT, Deans SG, Dorman HDJPm. Antioxidant and antimicrobial activity of Foeniculum vulgare and Crithmum maritimum essential oils. 2000;66(08):687-93.

151. Özbek H, Ugras S, Bayram I, Uygan I, Erdogan E, Öztürk A, et al. Hepatoprotective effect of Foeniculum vulgare essential oil: A carbon-tetrachloride induced liver fibrosis model in rats. 2004;31(1):9-17.

152. Zargari AJVI. Medicinal Plants Tehran University Publication. 1991.

153. Amini-Shirazi N, Hoseini A, Ranjbar A, Mohammadirad A, Khoshakhlagh P, Yasa N, et al. Inhibition of tumor necrosis factor and nitrosative/ oxidative stresses by Ziziphora clinopoides (Kahlioti); a molecular mechanism of protection against dextran sodium sulfate-induced colitis in mice. 2009;19(2):1839.

154. Naeini A, Khosravi A, Tadjbakhsh H, Ghazanfari T, Yaraee R, Shokri HJCcp. Evaluation of the immunostimulatory activity of Ziziphora tenuior extracts. 2010;19(5):459-63.

155. Yazdi FT, Mortazavi A, Koocheki A, Afsharian S, Behbahani BAJSJoM. Antimicrobial properties of plant extracts of Thymus vulgaris L., Ziziphora tenuior L. and Mentha Spicata L., against important foodborne pathogens in vitro. 2013;2(2):23-30.

156. Galli F, Azzi A, Birringer M, Cook-Mills JM, Eggersdorfer M, Frank J, et al. Vitamin E: Emerging aspects and new directions. 2017;102:16-36.

157. Padayatty SJ, Katz A, Wang Y, Eck P, Kwon O, Lee J-H, et al. Vitamin $\mathrm{C}$ as an antioxidant: evaluation of its role in disease prevention. 2003;22(1):18-35.

158. Sadeghi-Yarandi M, Karimi A, Ahmadi V, Sajedian AA, Soltanzadeh A, Golbabaei F. Cancer and non-cancer health risk assessment of occupational exposure to 1 , 3-butadiene in a petrochemical plant in Iran. Toxicology
Dispersive Liquid-Liquid Microextraction for the Determination of Salivary Melatonin as a Biomarker of Circadian Rhythm. 2018;73(10):966-72.

132. Al-Gnami SJJPBS. Effect of polyphenols which extracted from green tea in reduce toxic effects of cadmium sulfate in rats livers. 2014;9:53-8.

133. Chacko SM, Thambi PT, Kuttan R, Nishigaki IJCm. Beneficial effects of green tea: a literature review. 2010;5(1):13.

134. Hamden K, CARREA U S, Ayadi Marki F, Masmoudi $\mathrm{H}$, El Feki AJBr. Positive effects of green tea on hepatic dysfunction, lipid peroxidation and antioxidant defence depletion induced by cadmium. 2008;41(3):331-9.

135. Winiarska-Mieczan AJRT, Pharmacology. The potential protective effect of green, black, red and white tea infusions against adverse effect of cadmium and lead during chronic exposure-A rat model study. 2015;73(2):521-9.

136. Park E-H, Kahng J-H, Lee SH, Shin K-HJF. An antiinflammatory principle from cactus. 2001;72(3):28890.

137. Kuti JOJFc. Antioxidant compounds from four Opuntia cactus pear fruit varieties. 2004;85(4):527-33.

138. Moßhammer MR, Stintzing FC, Carle RJIFS, Technologies E. Development of a process for the production of a betalain-based colouring foodstuff from cactus pear. 2005;6(2):221-31.

139. Okada K, Wangpoengtrakul C, Tanaka T, Toyokuni S, Uchida K, Osawa TJTJon. Curcumin and especially tetrahydrocurcumin ameliorate oxidative stressinduced renal injury in mice. 2001;131(8):2090-5.

140. Bisht S, Feldmann G, Soni S, Ravi R, Karikar C, Maitra A, et al. Polymeric nanoparticle-encapsulated curcumin (“ nanocurcumin”): a novel strategy for human cancer therapy. 2007;5(1):3.

141. Hassani S, Sepand M, Jafari A, Jaafari J, Rezaee R, Zeinali $M$, et al. Protective effects of curcumin and vitamin $\mathrm{E}$ against chlorpyrifos-induced lung oxidative damage. 2015;34(6):668-76.

142. Piper JT, Singhal SS, Salameh MS, Torman RT, Awasthi YC, Awasthi SJTijob, et al. Mechanisms of anticarcinogenic properties of curcumin: the effect of curcumin on glutathione linked detoxification enzymes in rat liver. 1998;30(4):445-56.

143. Ruby AJ, Kuttan G, Babu KD, Rajasekharan K, Kuttan RJCl. Anti-tumour and antioxidant activity of natural curcuminoids. 1995;94(1):79-83.

144. Ajibola A, Chamunorwa JP, Erlwanger KHJN, metabolism. Nutraceutical values of natural honey and its contribution to human health and wealth. 2012;9(1):61.

145. Pyrzynska K, Biesaga MJTtiac. Analysis of phenolic 
salmon. 2015;10(3):e0119250.

171. Ackland ML, Michalczyk AJG, nutrition. Zinc deficiency and its inherited disorders-a review. 2006;1(1):41-9.

172. Russo AJN, insights $m$. Decreased zinc and increased copper in individuals with anxiety. 2011;4:NMI. S6349.

173. Maze P, editor Influences respectives des elements de la solution gmineral du mais. Annales de l'Institut Pasteur (Paris); 1914.

174. Vallee BL, Auld DSJB. Zinc coordination, function, and structure of zinc enzymes and other proteins. 1990;29(24):5647-59.

175. Miller WJ, Blackmon DM, Gentry R, Pitts W, Powell GJTJon. Absorption, excretion, and retention of orally administered zinc- 65 in various tissues of zinc-deficient and normal goats and calves. 1967;92(1):71-8.

176. Brown RS, Sander C, Argos PJFl. The primary structure of transcription factor TFIIIA has 12 consecutive repeats. 1985;186(2):271-4.

177. Prasad ASJJotACoN. Clinical and biochemical manifestations of zinc deficiency in human subjects. 1985;4(1):65-72.

178. Cunnane SJPif, science $n$. Role of zinc in lipid and fatty acid metabolism and in membranes. 1988;12(2):15188.

179. Keen CL, Ensunsa J, Watson M, Baly D, Donovan S, Monaco $\mathrm{M}$, et al. Nutritional aspects of manganese from experimental studies. 1999;20(2-3):213-23.

180. Erikson KM, Syversen T, Aschner JL, Aschner MJEt, pharmacology. Interactions between excessive manganese exposures and dietary iron-deficiency in neurodegeneration. 2005;19(3):415-21.

181. Fakhri-Bafghi MS, Ghasemi-Niri SF, Mostafalou S, Navaei-Nigjeh M, Baeeri M, Mohammadirad A, et al. Protective effect of selenium-based medicines on toxicity of three common organophosphorus compounds in human erythrocytes in vitro. 2016;17(4):740.

182. Navaei-Nigjeh $M$, Asadi H, Baeeri M, Pedram S, Rezvanfar MA, Mohammadirad A, et al. In vitro protection of human lymphocytes from toxic effects of chlorpyrifos by selenium-enriched medicines. 2015;18(3):284.

183. Kaur R, Sandhu HJEt, pharmacology. In vivo changes in antioxidant system and protective role of selenium in chlorpyrifos-induced subchronic toxicity in bubalus bubalis. 2008;26(1):45-8.

184. Amarowicz RJEJoLS, Technology. Lycopene as a natural antioxidant. 2011;113(6):675-7.

185. Yonar ME, Sakin FJPB, Physiology. Ameliorative effect of lycopene on antioxidant status in Cyprinus carpio during pyrethroid deltamethrin exposure. 2011;99(3):226-31. and Industrial Health. 2020:0748233720962238.

159. Milošević MD, Paunović MG, Matić MM, Ognjanović BI, Saičić ZSJEt, pharmacology. The ameliorating effects of selenium and vitamin $\mathrm{C}$ against fenitrothioninduced blood toxicity in Wistar rats. 2017;56:204-9.

160. Amara IB, Soudani N, Hakim A, Troudi A, Zeghal KM, Boudawara T, et al. Protective effects of vitamin E and selenium against dimethoate-induced cardiotoxicity in vivo: Biochemical and histological studies. 2013;28(11):630-43.

161. Narra MR, Rajender K, Reddy RR, Rao JV, Begum GJC. The role of vitamin $\mathrm{C}$ as antioxidant in protection of biochemical and haematological stress induced by chlorpyrifos in freshwater fish Clarias batrachus. 2015;132:172-8.

162. Raina R, Baba NA, Verma PK, Sultana M, Singh MJBter. Hepatotoxicity induced by subchronic exposure of fluoride and chlorpyrifos in Wistar rats: Mitigating effect of ascorbic acid. 2015;166(2):157-62.

163. Rajpoot DS, Prakash A, Mandil R, Rahal A, Garg SKJJoT, Environmental Health PA. Differential modulation of xenobiotic-metabolizing enzymes in rats following single and concurrent exposure to chlorpyrifos, arsenic, and ascorbic acid. 2013;76(24):1354-65.

164. Tripathi G, Shasmal JJE, safety e. Reparation of chlorpyrifos-induced impairment by thyroxine and vitamin C in fish. 2010;73(6):1397-401.

165. Aly N, Kawther E-G, Mahmoud F, El-Sebae AKJPB, Physiology. Protective effect of vitamin C against chlorpyrifos oxidative stress in male mice. 2010;97(1):712.

166. Uzunhisarcikli M, Kalender YJE, safety e. Protective effects of vitamins $\mathrm{C}$ and $\mathrm{E}$ against hepatotoxicity induced by methyl parathion in rats. 2011;74(7):21128.

167. El-Hack MEA, Mahrose K, Arif M, Chaudhry MT, Saadeldin IM, Saeed M, et al. Alleviating the environmental heat burden on laying hens by feeding on diets enriched with certain antioxidants (vitamin $\mathrm{E}$ and selenium) individually or combined. 2017;24(11):10708-17.

168. Verma RS, Mehta A, Srivastava NJPB, Physiology. In vivo chlorpyrifos induced oxidative stress: attenuation by antioxidant vitamins. 2007;88(2):191-6.

169. Saoudi M, Hmida IB, Kammoun W, Rebah FB, Jamoussi K, Feki AE. Protective effects of oil of Sardinella pilchardis against subacute chlorpyrifosinduced oxidative stress in female rats. Archives of environmental \& occupational health. 2018;73(2):12835.

170. Olsvik PA, Berntssen MH, Søfteland LJPo. Modifying effects of vitamin $\mathrm{E}$ on chlorpyrifos toxicity in Atlantic 
استفاده از تركيبات سرشار از مواد آنتى اكسيدان ...

2018;8:10.4172.

194. Abdel-Diam MM, Samak DH, El-Sayed YS, Aleya L, Alarifi S, Alkahtani SJES, et al. Curcumin and quercetin synergistically attenuate subacute diazinon-induced inflammation and oxidative neurohepatic damage, and acetylcholinesterase inhibition in albino rats. 2019;26(4):3659-65.

195. Abdollahzadeh Estakhri M, Shokrzadeh M, Jaafari MR, Mohammadi HJIJoBMS. Organ toxicity attenuation by nanomicelles containing curcuminoids: Comparing the protective effects on tissues oxidative damage induced by diazinon. 2019;22(1):17-24.

196. Khalifa FK, Alkhalf MIJJoKSU-S. Effects of black seed and thyme leaves dietary supplements against malathion insecticide-induced toxicity in experimental rat model. 2019.

197. Khan N, Mukhtar HJN. Tea Polyphenols in Promotion of Human Health. 2019;11(1):39.

198. Russo GL, Ungaro P. Epigenetic Mechanisms of Quercetin and Other Flavonoids in Cancer Therapy and Prevention. Epigenetics of Cancer Prevention: Elsevier; 2019. p. 187-202.

199. Callcott ET, Santhakumar AB, Luo J, Blanchard CLJJoAB. Therapeutic potential of rice-derived polyphenols on obesity-related oxidative stress and inflammation. 2018;16(4):255-62.

200. Giglio RV, Patti AM, Cicero AF, Lippi G, Rizzo M, Toth PP, et al. Polyphenols: potential use in the prevention and treatment of cardiovascular diseases. 2018;24(2):239-58.

201. Sakaki J, Melough M, Lee SG, Pounis G, Chun OK. Polyphenol-rich diets in cardiovascular disease prevention. Analysis in Nutrition Research: Elsevier; 2019. p. 259-98
186. Yonar MEJF, immunology s. The effect of lycopene on oxytetracycline-induced oxidative stress and immunosuppression in rainbow trout (Oncorhynchus mykiss, W.). 2012;32(6):994-1001.

187. Ural MŞJC. Chlorpyrifos-induced changes in oxidant/ antioxidant status and haematological parameters of Cyprinus carpio carpio: ameliorative effect of lycopene. 2013;90(7):2059-64.

188. Kofod DH, Jørs E, Varma A, Bhatta S, Thomsen JFJEh. The use of self-reported symptoms as a proxy for acute organophosphate poisoning after exposure to chlorpyrifos $50 \%$ plus cypermethrin $5 \%$ among Nepali farmers: a randomized, double-blind, placebocontrolled, crossover study. 2016;15(1):122.

189. Tam NT, Berg H, Van Cong NJES, Research P. Evaluation of the joint toxicity of chlorpyrifos ethyl and fenobucarb on climbing perch (Anabas testudineus) from rice fields in the Mekong Delta, Vietnam. 2018;25(14):13226-34.

190. Heikal T, Mossa A, Marei G, Abdel Rasoul MJJEAT. Cyromazine and chlorpyrifos induced renal toxicity in rats: the ameliorating effects of green tea extract. 2012;2(146):2161.

191. Fereidouni S, Kumar RR, Chadha VD, Dhawan DKJJob, toxicology m. Quercetin plays protective role in oxidative induced apoptotic events during chronic chlorpyrifos exposure to rats. 2019:e22341.

192. Sahinoz E, Aral F, Dogu Z, Koyuncu İ, Yuksekdag O. The Protective Effects of Curcumin on Organophosphate Insecticide Chlorpyrifos-Induced Oxidative Stress and DNA Damage in Oncorhynchus mykiss.

193. El-Wakf A, El-Habibi E, Barakat N, Attia A, Hussein A, Ali IJJCT. Cardiovascular toxic effects of chlorpyrifos: A possible protective role for pomegranate extracts. 